\title{
On fluid flow in a heterogeneous medium under nonisothermal conditions
}

\author{
D. W. Vasco, Lawrence Berkeley National Laboratory, Berkeley, CA 94720
}

\begin{abstract}
An asymptotic technique, valid in the presence of smoothly-varying heterogeneity, provides explicit expressions for the velocity of a propagating pressure and temperature disturbance. The governing equations contain nonlinear terms due to the presence of temperature-dependent coefficients and due to the advection of fluids with differing temperatures. Two cases give well-defined expressions in terms of the parameters of the porous medium: the uncoupled propagation of a pressure disturbance and the propagation of a fully coupled temperature and pressure disturbance. The velocity of the coupled disturbance or front, depends upon the medium parameters and upon the change in temperature and pressure across the front. For uncoupled flow, the semi-analytic expression for the front velocity reduces to that associated with a linear diffusion equation. A comparison of the asymptotic travel time estimates with calculations from a numerical simulator indicates reasonably good agreement for both uncoupled and coupled disturbances.
\end{abstract}

\section{Introduction}

Nonisothermal flow is a common occurrence in actual field settings. That is, there are variations in the temperature of fluids in the subsurface, for example due to the Earth's thermal gradient or due to seasonal effects [Grifoll et al., 2005]. These temperature variations are usually small enough that their effect on fluid properties can be safely ignored. In certain activities, such as geothermal production [Brownell et al., 1977; Mercer and Faust, 1979] or high-temperature waterflooding [Boberg, 1988], the differences in fluid temperature are so large that they must be accommodated when modeling such flow.

Fluid flow under nonisothermal conditions is typically more complicated than is isothermal fluid flow [Brownell et al., 1977; Bear and Corapcioglu, 1981; Noorishad et al., 1984; McTigue, 1986]. Nonisothermal flow, even under the simplest conditions in which matrix deformation is neglected, is governed by two coupled equations. Furthermore, two forms of nonlinearity are present in the governing equations. First, there are nonlinearities due to the temperature dependence of the coefficients in the governing equations. Second, there are explicit nonlinear terms in the mass and energy equations. These nonlinearities take the form of the scalar products of the pressure and temperature gradients. Due to the complexities of such coupled nonlinear equations with spatially-varying parameters, most investigators have turned to numerical methods in order to model nonisothermal flow [Noorishad et al., 1984]. Previous analytic or semi-analytic studies have been limited to rather restrictive situations, such as a homogeneous [Booker and Savvidou, 1984] or one-dimensional medium [O’Sullivan, 1981; Natale and Salusti, 1996; Doughty and Pruess, 1992], or linearized versions of the governing equations [McTigue, 1986].

In this paper I present a semi-analytic approach for modeling nonisothermal flow in a heterogeneous medium. The approach is based upon an asymptotic technique that has proven useful in modeling coupled linear processes [Vasco, 2009], as well as nonlinear processes such as two-phase flow [Vasco, 2004]. The asymptotic procedure assumes that the length scale of the heterogeneity is greater than the length scale of the propagating pressure and temperature disturbances. Here, I extend this approach to coupled, nonlinear processes in a three-dimensional heterogeneous medium. Away from layer boundaries and faults, the heterogeneity is assumed to vary in a smooth fashion but with an arbitrary magnitude. Specifically, the length scale of the heterogeneity is assumed to be longer than that of the propagating pressure and temperature disturbance. One use of such modeling is for efficient inversion of flow and temperature data. The techniques developed here are intended to be used with a numerical simulator, both to aid in the interpretation of results and as a method for the inverse modeling of observations. For example, the asymptotic formulation provides explicit semi-analytic expressions for model parameter sensitivies [Vasco et al., 2000; Vasco, 2008]. Though the specific formulation of Noorishad et al. [1984], modified along the lines of the passive reservoir model of Brownell et al. [1977], is used here, and the fluid is assumed to be incompressible, the approach is general and applicable to more comprehensive formulations. That is, the techniques can be applied to general systems of nonlinear, as well as linear, governing equations.

\section{Methodology}

\subsection{The Governing Equations for Nonisothermal Flow}

\subsubsection{The Conservation of Mass and Momentum}

I begin with the conservation of mass and momentum, as given in Noorishad et al. [1984]

$$
\frac{\partial \eta}{\partial t}-\frac{e}{\rho_{0}} \frac{\partial \rho_{l}}{\partial t}=\nabla \cdot\left[\frac{\rho_{l} K}{\rho_{0} \mu}\left(\nabla P+\rho_{l} \mathbf{Z}\right)\right]
$$

where $\eta$ is the equivalent fluid volume strain, $e$ the volumetric strain in the solid, $\rho_{l}$ the fluid density, $\rho_{o}$ a reference density, $K$ the permeability, $\mu$ the fluid viscosity, $P$ the fluid pressure, and $\mathbf{Z}=g \mathbf{z}$ the gravitational force vector. For this study I consider only volumetric changes due to local thermal and fluid pressure changes. That is, I will not consider stresses transmitted 
across the reservoir and through the overburden. In addition, it will be assumed that the stresses due to the overburden are not changing. For volumetric changes induced by fluid pressure and temperature changes I can rewrite equation (1) in the form

$$
\gamma_{P} \frac{\partial P}{\partial t}+\gamma_{T} \frac{\partial T}{\partial t}=\nabla \cdot\left[\frac{\rho_{l} K}{\rho_{0} \mu} \nabla P+\frac{\rho_{l}^{2} K}{\rho_{0} \mu} \mathbf{Z}\right]
$$

where $\gamma_{P}$ and $\gamma_{T}$ are functions of $\mathbf{x}$, and possibly pressure and temperature, given by

$$
\gamma_{P}=\phi\left(\beta_{l}-\beta_{s}+\frac{\alpha}{\phi}\right)
$$

where $\beta_{l}$ is compressibility coefficient for the fluid, $\beta_{s}$ is the compressibility coefficient for the solid, $\alpha$ is the compressibility coefficient of the porous matrix [de Marsily, 1986, p. 108], and

$$
\gamma_{T}=\phi\left(\frac{\partial \rho_{l}}{\partial T}\right)_{\rho_{0}}
$$

is the thermal expansion coefficient of the fluid. Both the fluid density $\rho_{l}$ and the fluid viscosity $\mu$ depend upon temperature while the permeability, $K$, is assumed to only depend upon position $\mathbf{x}$.

In the most general formulation, one would allow the fluid density and fluid viscosity to depend upon both the temperature and pressure. And the techniques presented in this paper are applicable when the density and viscosity also depend upon pressure. However, for no other reason then simplicity, I shall assume that the fluid density and the fluid viscosity only depend upon the temperature of the fluid. This assumption is a fairly good approximation for water under the conditions considered in the applications (Figure 1). For the pressures and temperatures in the numerical tests below, only the density varies as a function of pressure, and then by only a fraction of a percent. In contrast, the fluid viscosity is a fairly strong function of temperature, varying by a factor of 20 from 1 to 150 degrees C. Defining the coefficients

$$
\Upsilon_{P}(T)=\frac{\rho_{l}(T)}{\rho_{0} \mu(T)}
$$

and

$$
\Upsilon_{Z}(T)=\frac{\rho_{l}(T)^{2}}{\rho_{0} \mu(T)}
$$

I can write equation (2) more compactly as

$$
\gamma_{P} \frac{\partial P}{\partial t}+\gamma_{T} \frac{\partial T}{\partial t}=\nabla \cdot\left[\Upsilon_{P} K \nabla P+\Upsilon_{Z} K \mathbf{Z}\right]
$$

Before expanding the divergence of the quantity in brackets, I note that because $\Upsilon_{P}$ and $\Upsilon_{Z}$ only depend implicitly upon $\mathbf{x}$, through their dependence upon $T$, I can write their gradients as

$$
\nabla \Upsilon_{P}=\xi_{P T} \nabla T
$$

and

$$
\nabla \Upsilon_{Z}=\xi_{Z T} \nabla T
$$

where

$$
\xi_{P T}=\frac{\partial \Upsilon_{P}}{\partial T}
$$

is a measure of the sensitivity of the coefficient $\Upsilon_{P}=$ $\rho_{l} /\left(\rho_{0} \mu\right)$ to variations in the temperature of the fluid. A similar expression holds for $\xi_{Z T}$. Applying the divergence operator to the quantity in brackets on the right-hand-side of equation (7) results in a partial differential equation for the fluid pressure and temperature

$$
\begin{gathered}
\gamma_{P} \frac{\partial P}{\partial t}+\gamma_{T} \frac{\partial T}{\partial t}=\left[\Upsilon_{P} \nabla K+K \xi_{P T} \nabla T\right] \cdot \nabla P \\
+\left[\Upsilon_{Z} \nabla K+K \xi_{Z T} \nabla T\right] \cdot \mathbf{Z}+\Upsilon_{P} K \nabla \cdot \nabla P .
\end{gathered}
$$

\subsubsection{The Conservation of Energy}

The conservation of energy provides a second governing equation. Again, I am considering the situation in which the matrix deformation, transmitted through the overburden and the reservoir, may be neglected. That is the passive reservoir model of Brownell et al. [1977] which leads to an energy equation of the form

$$
\begin{gathered}
\eta \frac{\partial T}{\partial t}=\left[\nabla K_{T}-C_{\rho} \Upsilon_{P} K \nabla P\right] \cdot \nabla T-C_{\rho} \Upsilon_{Z} K \nabla T \cdot \mathbf{Z} \\
+K_{T} \nabla \cdot \nabla T
\end{gathered}
$$

where

$$
\eta=\phi \rho_{l} C_{l}+(1-\phi) \rho_{s} C_{s}
$$

is the volumetric heat capacity of the rock, a weighted average of the heat capacity of the liquid and the heat capacity of the solid,

$$
C_{\rho}=C_{l} \rho_{0}
$$

is the volumetric heat capacity of the liquid, and

$$
K_{T}=\phi K_{l}+(1-\phi) K_{s}
$$

is the thermal conductivity of the composite material, a weighted average of the liquid and solid conductivities [Carslaw and Jaeger, 1959; Crank, 1975]. These coefficients are defined in terms of the porosity $\phi$, the fluid $\left(\rho_{l}\right)$ and solid $\left(\rho_{s}\right)$ densities, the fluid and solid specific heat constants $C_{l}$ and $C_{s}$, and the fluid and solid thermal conductivities $K_{l}$ and $K_{s}$, respectively [Noorishad et al., 1984].

In formulating equation (12) I am assuming that the solid and fluid reach thermal equilibrium rapidly and a single temperature $T$ is sufficient to represent the thermal state of the solid and the fluid. As noted by de Marsily [1986], it has been shown that temperatures equilibrate in less than a minute for a medium with a grain size of less than $1 \mathrm{~mm}$. This assumption has been widely adopted in the modeling of fluid flow under nonisothermal conditions, for example in geothermal reservoirs [Brownell et al., 1977]. The modifications required in relaxing this assumption have been presented in a number of papers [Auriault and Royer, 1993; Moyne, 1997; Quintard et al., 1997; Hsiao and Advani, 1999; Sozer and Shyy, 2008]. The techniques discussed in this paper could be applied to these more general equations, introducing an additional governing equation and more complicated expressions.

\subsubsection{The System of Governing Equations}

I can consolidate the coefficients in equation (11) if I divide through by $K(\mathbf{x})$ and define

$$
\begin{aligned}
\zeta_{P} & =\frac{\gamma_{P}}{K} \\
\zeta_{T} & =\frac{\gamma_{T}}{K}
\end{aligned}
$$




$$
\lambda=-\ln K
$$

In doing so, I am assuming that the permeability is never zero. In fact, this must be true if one wishes to generate flow. The equations governing fluid flow in a nonisothermal medium are then

$$
\begin{gathered}
\zeta_{P} \frac{\partial P}{\partial t}+\zeta_{T} \frac{\partial T}{\partial t}=\left[\Upsilon_{P} \nabla \lambda+\xi_{P T} \nabla T\right] \cdot \nabla P \\
+\left[\Upsilon_{Z} \nabla \lambda+\xi_{Z T} \nabla T\right] \cdot \mathbf{Z}+\Upsilon_{P} \nabla \cdot \nabla P
\end{gathered}
$$

and

$$
\begin{gathered}
\eta \frac{\partial T}{\partial t}=\left[\nabla K_{T}-C_{\rho} \Upsilon_{P} K \nabla P\right] \cdot \nabla T-C_{\rho} \Upsilon_{Z} K \nabla T \cdot \mathbf{Z} \\
+K_{T} \nabla \cdot \nabla T
\end{gathered}
$$

two coupled, nonlinear, partial differential equations for $T(\mathbf{x}, t)$ and $P(\mathbf{x}, t)$. Note that there are other formulations of nonisothermal flow, with somewhat different terms present, and based on different assumptions [Brownell et al., 1977; Pinder, 1979; Bear and Corapcioglu, 1981]. However, the exact formulation of nonisothermal flow is not critical. The asymptotic techniques presented in this paper are general and can be applied to any of the corresponding governing equations.

\subsection{An Asymptotic Analysis of the Governing Equations: Propagation in a Medium with Smoothly- Varying Heterogeneity}

The governing equations (19) and (20) are rather complicated because they are nonlinear, of mixed character, and coupled partial differential equations with spatially-varying coefficients. Without some manner of simplification an analytic solution is certainly not possible. Because one goal of this work is to develop techniques for solving inverse problems, for example using the thermal front arrival time to say something about flow properties, accounting for heterogeneity is essential. However, due to the limited resolution of most inverse methods, in which a finite number of data are used to estimate a field of spatially-varying properties, one typically seeks models with smoothly-varying heterogeneity. Thus, I am most interested in modeling fluid flow in a medium with smoothly-varying properties. I should note that sharp boundaries, in the form of layering or a fault, are allowed as boundary conditions.

I can build the assumption of smoothly-varying heterogeneity into the modeling through a technique known as the method of multiple scales [Anile et al., 1993, p. 49]. This approach is suited to the construction of asymptotic solutions for a porous medium with heterogeneous, yet smoothlyvarying flow properties. The measure of smoothness is with respect to the length scales of the propagating temperature and pressure disturbances. In order to define this formally, I follow the approach of Anile et al. [1993] and denote the length scale of the front, eg. the distance over which the pressure and temperature changes from a background value to the value behind the front, by $l$. In addition, let $L$ denote the length scale of the heterogeneity within the medium. The smoothness of the medium is stipulated by the requirement that $L \gg l$. An asymptotic solution can be formulated in terms of the ratio of scale-lengths $\varepsilon=l / L$, where $\varepsilon \ll 1$. In order to isolate the scale of interest, I define the slow spatial coordinates

$$
\mathbf{X}=\varepsilon \mathbf{x}
$$

the scale over which many of the quantities of interest, such as the travel time and the amplitude will vary. Similarly, I

$$
\tau=\varepsilon t
$$

Intuitively, the assumption of smoothly-varying heterogeneity allows one to treat the propagating temperature and pressure disturbances as local fronts. Concepts associated with front propagation, such as the travel time, or phase, and the amplitude of the front may be employed. Furthermore, one also assumes that local changes in these quantities, front amplitude and phase, are due to local changes in the properties of the medium. Thus, one can follow the evolution of a given small segment of the front over time, leading to the idea of a trajectory or ray. Furthermore, the fields at a given location can be obtained by a summation over all trajectories connecting the observation point to the source point.

An asymptotic solution is a power series representation of the dependent variables, that is, the temperature and pressure. The form of the series is motivated by mathematical and physical studies of propagating fronts [Kline and Kay, 1965; Maslov and Omel'yanov, 2001]. In particular, there is an exponential phase term to model the propagation of the disturbance and successive amplitude terms to model changes in the magnitude of the disturbance. The formal power series is in terms of the scale variable $\varepsilon$. Because $\varepsilon$ is assumed to be small, the first few terms of the series are the most significant. The asymptotic solution for the temperature is represented as

$$
T\left(\mathbf{X}, \tau, \theta_{T}\right)=T_{b}(\mathbf{X}, \tau)+\int_{0}^{\tau} e^{\theta_{T}(\mathbf{X}, u)} \sum_{i=0}^{\infty} \varepsilon^{i} T_{i}(\mathbf{X}, u) d u
$$

where $T_{b}$ is the background temperature which may be a function of space and time, $\theta_{T}(\mathbf{X}, \tau)$ is a phase function that is related to the propagation of the thermal front, and $T_{i}(\mathbf{X}, \tau)$ is the i-th contribution to the amplitude of the temperature change. The temperature change contains both an explicit and an implicit dependence upon the spatial and temporal coordinates. The implicit dependence is through the function phase function $\theta_{T}(\mathbf{X}, \tau)$.

Due to the nature of the source most commonly used in injection and pumping, a constant rate function similar to a step, I have modified the representation by integrating over the temporal variable. That is, because a typical source in hydrology is a step-function in time, the time-integral of a more traditional impulsive source, I represent the solution as an integral over time of the traditional asymptotic solution. For an impulsive source-time function, one can remove the integral in equation (23) and work with the conventional form of the asymptotic expansion.

The change in pressure across the front has a similar representation

$$
P\left(\mathbf{X}, \tau, \theta_{P}\right)=P_{b}(\mathbf{X}, \tau)+\int_{0}^{\tau} e^{\theta_{P}(\mathbf{X}, u)} \sum_{i=0}^{\infty} \varepsilon^{i} P_{i}(\mathbf{X}, u) d u
$$

where $\theta_{P}(\mathbf{X}, \tau)$ is the phase function and $P_{i}(\mathbf{X}, \tau)$ are the amplitude functions. Note that the phase function for the pressure, $\theta_{P}$, can differ from the phase associated with the temperature, $\theta_{T}$, meaning that the temperature and pressure changes can move with different speeds. For example, an initial jump in pressure might propagate faster then a coupled pressure-thermal front. Later in the paper I will 
examine the situations in which the changes are both uncoupled and coupled and $\theta_{T}$ is equal to $\theta_{P}$.

The governing equations (19) and (20) can be rewritten in terms of the slow coordinates $\mathbf{X}$ and $\tau$. In order to do this, I first recast the partial derivatives in terms of $X_{i}, \tau$, and $\theta$. In doing so I make use of the relationships (21) and (22) between the fast and slow coordinates and the explicit and implicit dependence upon the independent variables. Thus, I can write the partial derivative with respect to time as

$$
\frac{\partial T}{\partial t}=\varepsilon \frac{\partial T}{\partial \tau}+\frac{\partial \theta_{T}}{\partial t} \frac{\partial T}{\partial \theta_{T}}
$$

because, from the definition of $\tau$, equation (22), $\partial \tau / \partial t=\varepsilon$. Similarly, I can express the derivative with respect to $x_{i}$ as

$$
\frac{\partial T}{\partial x_{i}}=\varepsilon \frac{\partial T}{\partial X_{i}}+\frac{\partial \theta_{T}}{\partial x_{i}} \frac{\partial T}{\partial \theta_{T}},
$$

and thus, in terms of the $\mathbf{X}$ coordinates, the gradient is given by

$$
\nabla_{x} T=\varepsilon \nabla_{X} T+\nabla \theta_{T} \frac{\partial T}{\partial \theta_{T}}
$$

Given these expressions for the differential operators, I can rewrite the governing equations in terms of the slow variables $\mathbf{X}$ and $\tau$. Each term will contain a factor composed of the scale parameter $\varepsilon$ raised to some power. Because we are assuming that the temperature and pressure changes vary over a length scale that is much less then that of the heterogeneity, $\varepsilon$ is assumed to be much smaller than one. Therefore, terms of low order in $\varepsilon$ will dominate in the governing equations. In Appendix A I write the governing equations in terms of the slow variables and retain terms of order $\varepsilon^{0} \sim 1$. To order $\varepsilon^{0}$ the governing equations take the form [see Appendix A, leading up to equations (A10) and (A18) for additional details]

$$
\begin{gathered}
\xi_{P T} \mathbf{s} \cdot \mathbf{p} \bar{T} \bar{P}+\alpha_{P} \bar{P}-\alpha_{T} \bar{T}=0 \\
C_{\rho} \Upsilon_{P} K \mathbf{p} \cdot \mathbf{s} \bar{T} \bar{P}-\beta_{T} \bar{T}=0
\end{gathered}
$$

where

$$
\bar{P}=P-P_{b}
$$

and

$$
\bar{T}=T-T_{b}
$$

are the changes in pressure and temperature that occur with the passage of the front, the vectors

$$
\mathbf{s}=\nabla \theta_{\mathbf{T}}
$$

and

$$
\mathbf{p}=\nabla \theta \mathbf{P}
$$

are the gradients of the temperature and pressure phase functions used in the series representations (23) and (24), the quantities $s=\left|\nabla \theta_{T}\right|$ and $p=\left|\nabla \theta_{P}\right|$ represent the magnitudes of these respective vectors. Finally, I have defined the coefficients,

$$
\begin{gathered}
\alpha_{P}=\Upsilon_{P} p^{2}-\zeta_{P} \frac{\partial \theta_{P}}{\partial t} \\
\alpha_{T}=\zeta_{T} \frac{\partial \theta_{T}}{\partial t}+\xi_{Z T} \mathbf{s} \cdot \mathbf{Z}
\end{gathered}
$$

and

$$
\beta_{T}=K_{T} s^{2}+C_{\rho} \Upsilon_{Z} K \mathbf{s} \cdot \mathbf{Z}-\eta \frac{\partial \theta_{T}}{\partial t} .
$$

There is an issue that needs to be addressed, the various coefficients in equations (28) and (29) are nonlinear functions of the temperature and pressure, and some are functions of the spatial coordinates as well. Consider, for example, $\Upsilon_{P}(T)$, which depends upon the temperature through it's dependence upon the fluid density and the fluid viscosity [see equation (6)]. I argue that the kinematics of the propagating pressure and temperature disturbances depend upon the background conditions in the medium. That is, the movement of propagating disturbance is controlled by the conditions at its leading edge. For it is the propagation of the leading edge that defines the sequence of front positions over time. Conditions behind the front primarily determine the steepness of the changes in pressure and temperature changes. To be sure this is an approximation that must be examined through comparison with numerical simulation. Therefore, I will assume that the coefficients in equations (28) and (29) are evaluated using the background pressure and temperature fields, $P_{b}$ and $T_{b}$, respectively.

\subsection{A Governing Equation for the Phase of the Propagating Pressure and Thermal Fronts}

In this sub-section I derive expressions for the phase. The phase is related to the slowness of the propagating thermal and pressure fronts. The slowness is the inverse of the propagation velocity. Such expressions may be used to compute the travel times of pressure and temperature disturbances through a heterogeneous medium.

Note that the two reduced governing equations (28) and (29) are polynomial equations in the amplitudes $\bar{P}$ and $\bar{T}$. It is well known, from algebraic geometry, that a system of polynomial equations has a non-trivial solution if the resultant, a determinant in the coefficients and a subset of the variables, vanishes [Cox et al., 1998, p. 71]. The conditions for a non-trivial solution of a system of governing equations is often used to find the slowness values associated with modes of propagation in a medium [Kratsov and Orlov, 1990]. However, in the case of equations (28) and (29) it is simpler to work directly with the algebraic expressions rather than with the resultant. In particular, I can use the second equation, equation (29), to solve for the product term $\mathbf{p} \cdot \mathbf{s} \bar{T} \bar{P}$

$$
\mathbf{p} \cdot \mathbf{s} \bar{T} \bar{P}=\frac{\beta_{T} \bar{T}}{C_{\rho} \Upsilon_{P} K}
$$

This expression may be substituted into equation (28) to arrive at

$$
\left(\frac{\xi_{P T} \beta_{T}}{C_{\rho} \Upsilon_{P} K}-\alpha_{T}\right) \bar{T}+\alpha_{P} \bar{P}=0 .
$$

Assuming that the amplitude of the temperature and pressure changes $\bar{T}$ and $\bar{P}$ are not simultaneously zero, that at least one disturbance is propagating, equation (38) specifies those values of $s$ and $p$ for which equations (28) and (29) have solutions. The character of equation (38) is clearer if I write it explicitly in terms of $s$ and $p$ and group terms according to their degree. The result is

$$
\begin{gathered}
\Upsilon_{P} \bar{P} p^{2}+\frac{\xi_{P T} K_{T} \bar{T}}{C_{\rho} \Upsilon_{P} K} s^{2} \\
+\left(\frac{\xi_{P T} \Upsilon_{Z}}{\Upsilon_{P}}-\xi_{Z T}\right) \bar{T} g \cos \left(\nu_{T}\right) s
\end{gathered}
$$




$$
-\left(\zeta_{P}+\frac{\xi_{P T} \eta}{C_{\rho} \Upsilon_{P} K}\right) \bar{T} \frac{\partial \theta_{T}}{\partial t}-\zeta_{P} \bar{P} \frac{\partial \theta_{P}}{\partial t}=0
$$

where $\nu_{T}$ signifies the angle between the gravitational force vector and the phase gradient vector $\mathbf{s}$. If $\bar{P}$ and $\bar{T}$ are known, for example from a numerical simulation, then equation (39) is a single quadratic equation for the two unknowns, $p$ and $s$. Thus, in the most general situation, it is not possible to determine $p$ and $s$ independently. However, there are situations that are of physical interest where one can find expressions for $p$ and $s$. One is the propagation of a coupled pressure and temperature disturbance. Another involves the propagation of a pressure disturbance well in advance of the advected hot or cold fluid. The first case, the propagation of a coupled pressure and temperature front, is of most interest and will be treated in the next section in some detail. The second situation, uncoupled pressure propagation will be discussed as a special case in the section on Applications.

\subsubsection{The Propagation of a Coupled Pressure and} Thermal Front

An advecting temperature change leads to a change in flow properties, most significantly a change in fluid viscosity, resulting in a pressure change across the thermal front. Because the temperature and pressure fronts propagate together, the pressure disturbance is initiated by the change in temperature, the phase functions are equal, $\theta=\theta_{P}=\theta_{T}$ and similarly for the phase vectors $\mathbf{p}=\mathbf{s}$. Thus, equation (39) is rewritten with $p=s$

$$
\begin{gathered}
{\left[\Upsilon_{P} \bar{P}+\frac{\xi_{P T} K_{T}}{C_{\rho} \Upsilon_{P} K} \bar{T}\right] p^{2}} \\
+\left[\left(\frac{\Upsilon_{Z}}{\Upsilon_{P}} \xi_{P T}-\xi_{Z T}\right) \bar{T}\right] g \cos \left(\nu_{P}\right) p \\
=\left[\zeta_{P} \bar{P}+\left(\zeta_{T}+\frac{\eta \xi_{P T}}{C_{\rho} \Upsilon_{P} K}\right) \bar{T}\right] \frac{\partial \theta}{\partial t} .
\end{gathered}
$$

Defining

$$
\Delta=\frac{\xi_{P T}}{C_{\rho} \Upsilon_{P} K}
$$

I can define the coefficients

$$
\Gamma=\frac{\left[\rho_{l} \xi_{P T}-\xi_{Z T}\right] g \bar{T}}{\Upsilon_{P} \bar{P}+K_{T} \Delta \bar{T}}
$$

and

$$
\Omega=\frac{\zeta_{P} \bar{P}+\left(\zeta_{T}+\eta \Delta\right) \bar{T}}{\Upsilon_{P} \bar{P}+K_{T} \Delta \bar{T}}
$$

and the quadratic equation (40) becomes

$$
p^{2}+\Gamma \cos \left(\nu_{P}\right) p-\Omega \frac{\partial \theta}{\partial t}=0
$$

Equation (44) is the most general expression for the slowness of a propagating coupled thermal and pressure front. In the formulation, I allow for temperature-sensitive fluid density and fluid viscosity. Furthermore, the flow properties of the medium are allowed to vary spatially. Gravitational forces are also included, rendering the slowness anisotropic, that is, the slowness depends upon the propagation direction of the front. Note that even if $\zeta_{T}$ is zero, there is no explicit pore expansivity in equation (19), the slowness still depends upon the temperature $(T)$ through the dependence of the fluid properties upon temperature, due to the coefficients $\xi_{P T}$ and $\xi_{Z T}$
One can say a bit more about the coefficient $\Gamma$ which represents the gravitational term, and gives rise to convective motion. Using the fact that $\xi_{P T}$ and $\xi_{Z T}$ represent the sensitivity of $\Upsilon_{P}$ and $\Upsilon_{Z}$ to variations in temperature [see equation (10)], I can write $\Gamma$ as

$$
\Gamma=\left[\rho_{l} \frac{\partial}{\partial T}\left(\frac{\rho_{l}}{\rho_{0} \mu}\right)-\frac{\partial}{\partial T}\left(\frac{\rho_{l}^{2}}{\rho_{0} \mu}\right)\right] \frac{g \bar{T}}{\Upsilon_{P} \bar{P}+K_{T} \Delta \bar{T}} .
$$

If the fluid density does not depend upon the temperature then $\Gamma$ vanishes. Thus, the convective term vanishes if the density of the fluid does not depend upon temperature. This fact follows from the initial assumption of an incompressible fluid, in which density of the fluid does not depend upon the fluid pressure. If one were to relax that assumption, a convective term would remain even if the fluid density did not depend upon the temperature. As indicated in Figure 1, the density of water has a moderate temperature-dependence, varying by roughly $10 \%$ as the temperature varies by 150 degrees.

\subsection{The Computation of the Phase and the Construction of a Trajectory-Based Solution}

The equations for the phase, as given by the quadratic equation (44), can be used to compute trajectories on which solutions can be defined. Thus, as for the ray-based methods that underlie electromagnetic [Kline and Kay, 1965; Bouche et al., 1997] and elastic [Karal and Keller, 1959; Chapman, 2004] wave propagation, the asymptotic approach produces a trajectory-based solution. Such techniques provide a foundation for efficient imaging algorithms, such as ray-based tomography [Iyer and Hirahara, 1993]. As the methods describe next, direct numerical integration and the method of characteristics, are general methods, they are applicable to the case in which gravity is important. For example, there are modifications to the numerical solvers for scalar partial differential equations in an anisotropic medium that can be applied to equation (44) [Qian and Symes, 2001]. Similarly, the method of characteristics can be used for general equations in which the slowness depends upon the direction of propagation [Courant and Hilbert, 1962, p. 106; Cerveny, 1972]. Because $p$ is the magnitude of the slowness vector, given by $\mathbf{p}=\nabla \theta$, I can write equation (44) as

$$
\nabla \theta \cdot \nabla \theta+\Gamma \nabla \theta \cdot \mathbf{Z}-\Omega \frac{\partial \theta}{\partial t}=0 .
$$

Equation (46) is a non-linear, first-order, partial differential equation for the phase function. It is an example of a class of equations [Lanczos, 1986, p. 229], that are instrumental in physics and applied mathematics. Equations of the this type also appear when modeling the propagation of an interface [Sethian, 1999].

\subsubsection{Direct Numerical Integration}

The most direct approach for solving equation (46) is a numerical technique, such as a level set method [Sethian, 1999]. Level set methods are efficient finite-difference schemes which converge to viscosity solutions of first-order partial differential equations, solutions of equation (46) augmented by a viscosity term for stabilization [Crandall and Lions, 1983; Crandall et al., 1984]. Using stable finitedifference schemes developed for hyperbolic conservation laws [LeVeque, 1992], one can solve the first-order, scalar, non-linear differential equation (46), augmented by a viscosity term, in an efficient and robust fashion [Sethian, 1990]. The fact that the gravitational term results in preferential movement in the direction of $\mathbf{Z}$ presents no fundamental difficulty. There are a number of implementations of finite-difference-based algorithms for anisotropic propagation, commonly used in seismic wave propagation in an 
anisotropic Earth [Lecomte, 1993; Eaton, 1993; Qian and Symes, 2001; Soukina et al., 2003]. Preferential propagation in a horizontal or vertical direction is also a factor in anisotropic crystal growth and finite-difference schemes have been used to model such propagation [Sethian and Strain, 1992].

\subsubsection{The Method of Characteristics}

Though a numerical approach is efficient and relatively easy to implement, there is an alternative classical approach that is efficient, provides some insight, and under some circumstances, leads to semi-analytic expressions for the phase. There is an extensive body of literature devoted to this approach that one can draw upon for insight and guidance. For example, there is a well-established method for solving firstorder, partial differential equations, motivated by geometrical arguments, the method of characteristics [Courant and Hilbert, 1962]. Using this approach one finds that equation (46) is equivalent to a system of first-order ordinary differential equations, the characteristic equations. These comprise equations for a trajectory along which a semi-analytic solution is defined. To see this, I first write equation (46) as

$$
F\left(\mathbf{x}, t, \theta, \mathbf{p}, \frac{\partial \theta}{\partial t}\right)=0
$$

where $F$ is the Hamiltonian function, given by

$$
F\left(\mathbf{x}, t, \theta, \mathbf{p}, \frac{\partial \theta}{\partial t}\right)=\mathbf{p} \cdot \mathbf{p}+\Gamma(\mathbf{x}, t, \theta) \mathbf{p} \cdot \mathbf{Z}+\Omega(\mathbf{x}, t, \theta) \frac{\partial \theta}{\partial t} .
$$

The system of characteristic equations corresponding to equation (47) is

$$
\begin{gathered}
\frac{d x_{i}}{d s}=\frac{\partial F}{\partial p_{i}} \\
\frac{d p_{i}}{d s}=-\frac{\partial F}{\partial x_{i}}-\frac{\partial F}{\partial \theta} p_{i}
\end{gathered}
$$

and

$$
\frac{d \theta}{d s}=\frac{\partial F}{\partial p_{i}} p_{i}
$$

[Courant and Hilbert, 1962, p. 106]. For the Hamiltonian function given by equation (48), the first two sets of characteristic equations are

$$
\begin{gathered}
\frac{d \mathbf{x}}{d s}=2 \mathbf{p}+\Gamma \mathbf{Z} \\
\frac{d \mathbf{p}}{d s}=-\nabla \Gamma \mathbf{p} \cdot \mathbf{Z}-\frac{\partial \Gamma}{\partial \theta} \mathbf{p} .
\end{gathered}
$$

The trajectory $\mathbf{x}(s)$ is calculated by solving the system of ordinary differential equations [Courant and Hilbert, 1962; Kravtsov and Orlov, 1990]. The development of algorithms for constructing the trajectories, or rays, is still an area of active research [Ruger, 2004].

\subsubsection{When the Phase Function is Separable}

In certain circumstances, the differential equation (46) is separable. There are two primary conditions for this: the vanishing of the gravitational term $\Gamma(\mathbf{x}, t, \theta)$, and the seperablity of the coefficient $\Omega(\mathbf{x}, t, \theta)$. The function $\Omega(\mathbf{x}, t, \theta)$ is said to be separable if it can be written as the product of two functions, the first function only depends upon $\mathbf{x}$ while the second only depends upon $t$ :

$$
\Omega(\mathbf{x}, t, \theta)=\Omega_{x}(\mathbf{x}) \Omega_{t}(t) .
$$

The function $\Omega(\mathbf{x}, t, \theta)$ is separable if the temperature and pressure disturbances display the same time-dependence. In that case one may derive an exact solution, as shown in Appendix B. The resulting phase function is of the form [see equation (B17)]

$$
\theta(\mathbf{x}, t)=-\frac{\sigma^{2}(\mathbf{x})}{4 t}
$$

where

$$
\sigma(\mathbf{x})=\int \sqrt{\Omega} d s
$$

and $\Omega(\mathbf{x})$ is given by equation (43).

\subsection{A Zeroth-Order Solution for Temperature and Pressure}

Once the phase function $\theta$ has been determined one can construct approximate solutions for the temperature and pressure variations. For example, the lowest-order representations of the solutions are obtained from the series (23) and (24) by retaining only the zeroth-order terms

$$
\bar{T}(\mathbf{X}, \tau, \theta)=\int_{0}^{\tau} e^{\theta(\mathbf{X}, u)} T_{0}(\mathbf{X}, u) d u
$$

for the temperature and

$$
\bar{P}(\mathbf{X}, \tau, \theta)=\int_{0}^{\tau} e^{\theta(\mathbf{X}, u)} P_{0}(\mathbf{X}, u) d u
$$

for the pressure. Here I am assuming that the temperature and pressure disturbances are coupled and propagate together, so that $\theta_{P}=\theta_{T}=\theta$. The integral appears in these expressions because I am assuming a source term that is a step-function function in time. The step-function source term represents the rapid onset of pumping or injection followed by a steady rate of flow. Such sources are frequently used and are necessary in order to generate sufficient flow at some distance from a well. For amplitude terms that are of one sign, the integral forms (57) and (58) generate monotonic variations in pressure and temperature for a given propagating front.

As noted by Virieux et al. [1994], and elaborated in Vasco et al. [2000] and Vasco and Finsterle [2004], it is fruitful to consider the time derivative of transient fields that are generated from a step-function source. By looking at the time derivative, the transient propagation of flow-related disturbances, such as pressure and temperature variations, is often more apparent. Furthermore, it is possible to define a quantity akin to an arrival time, a quantity that can be related to the flow properties of the medium [Virieux et al., 1994]. Such a relationship can be used to image the flow properties in the vicinity of an active well [Vasco et al., 2008]. If I consider the time derivatives of the zeroth-order asymptotic expressions I obtain

$$
\frac{\partial \bar{T}(\mathbf{X}, \tau, \theta)}{\partial \tau}=e^{\theta(\mathbf{X}, \tau)} T_{0}(\mathbf{X}, \tau)
$$

for the temperature and

$$
\frac{\partial \bar{P}(\mathbf{X}, \tau, \theta)}{\partial \tau}=e^{\theta(\mathbf{X}, \tau)} P_{0}(\mathbf{X}, \tau)
$$

for the pressure. These forms are useful when relating the phase $\theta$ to some measure of the arrival time of a propagating temperature and pressure disturbance.

As an example, consider the solution for a propagating pressure disturbance. Motivated by the form of the asymptotic solutions of the equation governing pressure in a heterogeneous medium [Vasco et al., 2000], I shall assume that 
the amplitude function $P_{0}(\mathbf{X}, \tau)$ may be written in a partitioned form. Furthermore, again based upon the solution of the linear diffusion equation, I shall assume a power-law time dependence, resulting in an amplitude function of the form

$$
P_{0}(\mathbf{X}, \tau)=\tau^{-a_{P}} \bar{P}_{0}(\mathbf{X})
$$

where $\bar{P}_{0}(\mathbf{X})$ is the function accounting for the spatial variation of the amplitude of the pressure change.

As discussed in Virieux et al. [1994] and Vasco et al. [2000], for an impulsive source, one measure of the arrival time of the temperature and pressure disturbances is the time at which the peak values are observed at a particular location. Because the source is generated by a step-function, I shall work with the time derivatives of the pressure and temperature fields. Thus, I will be concerned with the moment at which the time derivative of $\partial \bar{P} / \partial t$ reaches a maximum, that is, when

$$
\frac{\partial^{2} \bar{P}(\mathbf{X}, \tau, \theta)}{\partial t^{2}}=0
$$

Differentiating the expression (60) with respect to time, and accounting for the particular form of the amplitude term $\bar{P}_{0}$, equation (61), yields

$$
\frac{\partial^{2} \bar{P}}{\partial t^{2}}=t^{-a_{P}} e^{\theta}\left[\frac{\partial \theta}{\partial t}-\frac{a_{P}}{t}\right] \bar{P}_{0} .
$$

The peak value is attained when the quantity in square brackets vanishes, or when,

$$
\frac{\partial \theta}{\partial t}=\frac{a_{P}}{t_{\text {peak }}}
$$

where $t_{\text {peak }}$ is the time as which the peak temperature is obtained.

\section{Applications}

Here I shall consider examples involving the flow of a high-temperature aqueous fluid into a reservoir. One of the examples involves the propagation of uncoupled pressure changes in a homogeneous medium. This example demonstrates that the expression (40), given above, reduces to the conventional expression for transient fluid flow. The next example illustrates some important aspects of nonisothermal coupled flow. Finally, I shall examine flow in a heterogeneous medium.

In all cases I will simplify matters by assuming that the reservoir is thin (one meter in thickness) horizontal, essentially two-dimensional, so that gravitational effects are negligible. That is, because the flow is always perpendicular to $\mathbf{Z}$, the linear terms in $p$ in equations (39) and (46) vanish. Including gravity does not involve any fundamental difficulties other than the additional work required to compute trajectories in a medium with anisotropic slowness, as noted above.

The working fluid is liquid water, subject to sufficient pressure that it remains in a fluid state. Because the thermal-physical properties of water are known, the parameters and coefficients that appear in the governing equations (19) and (20) are well constrained. In particular, the pressure and temperature dependence of the fluid density and fluid viscosity are characterized by laboratory studies. In Figure 1 I have plotted the fluid density and viscosity of water as functions of the fluid pressure and temperature. The range of pressure and temperature variations reflects the conditions of the numerical experiments presented below. The background pressure, before the injection of hot water, is $5.0 \mathrm{MPa}$, and all pressures are references to that value. The injection of hot water is a model for energy storage in an aquifer, or the re-injection of geothermal water in a shallower part of the reservoir [Bear and Corapcioglu, 1981]. Such hot water injection is also used in secondary oil recovery [Boberg, 1988].

The variation of density and viscosity with temperature and pressure, shown in Figure 1, indicates that the density will not vary dramatically for the test cases. For example, the primary variation in density is as a function of temperature, and for the temperature range considered, between 25 and $150{ }^{\circ} \mathrm{C}$, the maximum density variation is roughly 8 $\%$. Furthermore, neither the density nor the viscosity vary significantly as a function of the fluid pressure. The primary variation with pressure occurs in the fluid density at temperatures below the background temperature of $25^{\circ} \mathrm{C}$. Even then, the density variation over the range of pressures is less then $1 \%$. The most significant variation in fluid properties is the change in viscosity as a function of temperature (Figure 1). The viscosity varies by roughly an order of magnitude between $25{ }^{\circ} \mathrm{C}$ and $150{ }^{\circ} \mathrm{C}$.

The overall geometry of the experiment consists of an injector in the center of the grid and an observation well to the northwest of the injector, as portrayed in Figure 2. At a particular time, which is taken as the origin of the time scale, a high-temperature fluid, with an enthalpy of $7.0 \times 10^{5}$ $\mathrm{J} / \mathrm{kg}$, is introduced into the porous medium at a constant flow rate of $10.0 \mathrm{~kg} / \mathrm{s}$. The fluid pressure and temperature variations as a function of space and time are computed using the numerical simulator TOUGH2 [Pruess et al., 1999]. The porous layer was sub-divided into a grid of 50 by 50 cells. Each cell in the grid was $100 \mathrm{~m}$ by $100 \mathrm{~m}$ laterally, and $1 \mathrm{~m}$ thick. The pressure and temperature variations after 4207 Days of injection are shown in Figure 2. In this figure the pressure scale signifies change in fluid pressure from a background value of 5.0 MPa. The edge of the grid is assumed to be held at a constant pressure and the heat capacity of the boundary grid blocks is taken to be an extremely large number. Note that, due to grid and boundary effects, the pressure and temperature variations do not display the rotational symmetry inherent in the problem. With a finer discretization and a larger mesh one could reduce these effects. However, that would increase the computation time required for the simulation of 45,000 days of injection.

\subsection{Propagation in a Homogeneous Medium}

First, consider flow in a homogeneous medium with a porosity $(\phi)$ of $10 \%$, a permeability $(K)$ of $2.0 \times 10^{-13} \mathrm{~m}^{2}$. The density of the solid matrix $\left(\rho_{s}\right)$ is $2650 \mathrm{~kg} / \mathrm{m}^{3}$, the specific heat of the matrix $\left(C_{l}\right)$ is $920.0 \mathrm{~J} / \mathrm{kg}^{\circ} \mathrm{C}$, the thermal conductivity $\left(K_{s}\right)$ is $2.51 \mathrm{~W} / \mathrm{m}^{\circ} C$, and the pore compressibility $\left(\gamma_{P}\right)$ is $1.5 \times 10^{-7} \mathrm{~Pa}^{-1}$. The simulated temperature and pressure variations associated with the observation well are shown in Figure 3. For comparison, in the left panel of Figure 3, I have also plotted the pressure associated with the injection of a fluid of the same temperature as the in situ fluid (labeled Isothermal). Note that, when the problem is isothermal, the pressure increases monotonically with the length of injection. When a high-temperature fluid is injected, the pressure initialy increases as in the isothermal case, but after about 3000 days the pressure decreases with time. The pressure decrease occurs as the temperature rises due to the arrival of the hot water at the observation well. Thus, for a nonisothermal injection there is a pressure increase due to the introduction of a mass of fluid and a pressure decrease due to the temperature-induced changes in the fluid properties. As noted above, we can use the expression for slowness [equation (40)] to examine these two cases in turn. 


\subsubsection{Uncoupled Pressure Propagation}

In this case there is no temperature change associated with the advancing change in pressure, and $\bar{T}$ is zero in equation (40). If there are no thermal or gravitational effects, the equation governing the phase is separable. This isothermal process leads to uncoupled pressure propagation, and equation (44) reduces to

$$
p^{2}=\frac{\zeta_{P}}{\Upsilon_{P}} \frac{\partial \theta}{\partial t}=0
$$

where the coefficients $\zeta_{P}$ and $\Upsilon_{P}$ only depend upon the spatial coordinates $\mathbf{x}$. The resulting partial differential equation for $\theta$, obtained by noting that $\mathbf{p}=\nabla \theta$, is separable and the results from Appendix B can be used. Therefore, I can work with a phase function of the form given in equation (B17)

$$
\theta=-\frac{\sigma^{2}}{4 t}
$$

Then, the zeroth-order expression for the time derivative of pressure, equation (60), is given by

$$
\frac{\partial \bar{P}(\mathbf{X}, \tau, \theta)}{\partial t}=t^{-a_{P}} \exp \left(-\frac{\sigma^{2}}{4 t}\right) \bar{P}_{0}(\mathbf{X}),
$$

where I have assumed that the amplitude function can be written in the separable form (61). In this case the condition for the peak of the pressure derivative, the vanishing of the second derivative of the pressure with respect to time [see equations (62) and (63)] gives

$$
\frac{\partial \theta}{\partial t}=\frac{\sigma^{2}}{4 t^{2}}
$$

Equation (64) provides a relationship between the arrival time of the peak and $\sigma$

$$
t_{\text {peak }}=\frac{\sigma^{2}}{4 a_{P}}
$$

or, using the definition of $\sigma$ given by equation (B16) in Appendix B,

$$
\sqrt{t_{\text {peak }}}=\frac{1}{2 \sqrt{a_{P}}} \int \sqrt{\Omega} d s
$$

Because the temperature change can be neglected, $\bar{T}$ vanished in equation (43), and the expression (69) reduces to

$$
\begin{aligned}
& \sqrt{t_{\text {peak }}}=\frac{1}{2 \sqrt{a_{P}}} \int \sqrt{\frac{\zeta_{P}}{\Upsilon_{P}}} d s \\
& =\frac{1}{2 \sqrt{a_{P}}} \int \sqrt{\frac{\gamma_{P}}{K} \frac{\rho_{0} \mu}{\rho_{l}}} d s
\end{aligned}
$$

where $\gamma_{P}$ is the coefficient of the time derivative of pressure in the governing equation (6), given by equation (3). If $\beta_{s}$ and $\alpha$ are much smaller than $\beta_{l}$ then $\gamma_{P}$ reduces to $\gamma_{P}=\phi \beta_{l}$ and the expression for $p^{2}$, equation (70) becomes

$$
\sqrt{t_{\text {peak }}}=\frac{1}{2 \sqrt{a_{P}}} \int \sqrt{\frac{\phi \beta_{l} \rho_{0}}{k}} d s
$$

where

$$
k=\frac{K \rho_{l}}{\mu} .
$$

Equation (71) is the expression for the square root of the travel time of a pressure disturbance propagating in a medium with smoothly-varying heterogeneity, the solution of a linear diffusion equation [Vasco et al., 2000].

The uncoupled transient pressure variation occurs well before the advected high-temperature fluid has arrived at a specific location. For example, consider Figure 3, the pressure and temperature variations simulated at the observation well. The location of the observation well is indicated by the open circle in Figure 2. At this location the temperature does not start increasing unil some time after 2000 days. However, there is a rapid build-up of pressure prior to that time. In Figure $4 \mathrm{I}$ plot the pressure increase as a function of time for the case of isothermal and nonisothermal flow. The pressure variation is shown for the first 500 days of injection, well before the arrival of the advected hot water. As noted by Vasco et al. [2000], for a constant flow rate, a step-function source, it is difficult to determine the 'arrival time' of a pressure disturbance. In the case of a step-function source, it is easier to work with the derivative of the pressure with respect to time and to compute an arrival time based upon the peak of the time derivative. In the right panel of Figure 4 I plot the time derivative of the fluid pressure as a function of time. Note that both the isothermal and nonisothermal injections have peaks at roughly 235 days.

The similarity of the transient pressure disturbance to a propagating front is clearer if one examines a sequence of 'snapshots' of the derivative of the pressure with respect to time. That is, I take the time series associated with each cell of the simulation grid and differentiate it with respect to time. I then normalize the time derivatives in each grid block by the peak value obtained in the respective grid block. In Figure 5 I have plotted three such 'snapshots' at 20, 98, and 446 days. The maximum values of the derivatives form a ring around the injection well and propagate outward over time. By recording the time at which the derivative is a maximum in each grid block, I can compute the arrival time of the transient pressure disturbance. An analytic expression relating the phase $\theta_{P}$ to the arrival time of the peak derivative was given in Virieux et al.[1994] and Vasco et al. [2000]. The travel time, calculated from the peak of the derivative in each grid block, is shown in Figure 6.

In the panel on the left in Figure 12 I plot the travel times for uncoupled pressure propagation, as calculated from the expression for slowness, equation (71) (Asymptotic), and estimated from the output of the TOUGH2 numerical simulator (Numeric). There is excellent agreement at the near and intermediate distances. The agreement degrades slightly at the longer offsets, perhaps due to the interaction with the boundary of the grid, which lies $2.5 \mathrm{~km}$ away from the injector. The constant pressure boundary condition is likely to have some influence as the edge of the grid is approached.

3.1.2. The Coupled Propagation of Temperature and Pressure Changes

Next I consider the propagation of a coupled temperature and pressure front, where the slowness is given by equation (44) with no gravitational term

$$
p^{2}-\Omega(\mathbf{x}, t, \theta) \frac{\partial \theta}{\partial t}=0
$$

with $\Omega$ given by equation (43):

$$
\Omega=\frac{\zeta_{P} \bar{P}+\left(\zeta_{T}+\eta \Delta\right) \bar{T}}{\Upsilon_{P} \bar{P}+K_{T} \Delta \bar{T}}
$$

Expression (74) is complicated and the temperature and pressure changes can both slow down and speed up the propagation of the coupled front. The parameters used to compute the slowness were identical to those used above: a porosity $(\phi)$ of $10 \%$, a permeability $(K)$ of $2.0 \times 10^{-13} \mathrm{~m}^{2}$. 
The density of the solid matrix $\left(\rho_{s}\right)$ is $2650.0 \mathrm{~kg} / \mathrm{m}^{3}$ while that of the liquid $\left(\rho_{l}\right)$ is $997.0 \mathrm{~kg} / \mathrm{m}^{3}$, the specific heat of the matrix $\left(C_{s}\right)$ is $920.0 \mathrm{~J} / \mathrm{kg}^{\circ} \mathrm{C}$, the specific heat of the liquid $\left(C_{l}\right)$ is $4180.0 \mathrm{~J} / \mathrm{kg}^{\circ} \mathrm{C}$, the thermal conductivity of the solid $\left(K_{s}\right)$ is $2.51 \mathrm{~W} / \mathrm{m}^{\circ} C$, the thermal conductivity of the liquid $\left(K_{l}\right)$ is $2.51 \mathrm{~W} / \mathrm{m}^{\circ} C$, and the pore compressibility $\left(\gamma_{P}\right)$ is $1.5 \times 10^{-7} \mathrm{~Pa}^{-1}$. The partial derivative of $\Upsilon_{P}$ with respect to the temperature, where $\Upsilon_{P}=\rho_{l} / \rho_{0} \mu$ is calculated by numerical differentiation, with a value of approximately 25.6 $(P a-s)^{-1}\left({ }^{o} C\right)^{-1}$. The temperature dependence of $\mu$ is obtained from routines contained in the TOUGH2 simulation package. The reference density $\rho_{0}$ is taken to be the liquid density $\rho_{l}$ so that the ratio $\rho_{l} / \rho_{0}$ is always unity. The values of the jumps in pressure $(\bar{P})$ and temperature $(\overline{\bar{T}})$ are computed from the results of the TOUGH2 simulation and have average values of $5.0 \mathrm{MPa}$ and $106{ }^{\circ} \mathrm{C}$, respectively. In Appendix C I indicate how phase-dependent terms of $\vec{T}$ and $\bar{P}$ can cancel for an impulsive or a step-function source.

For the example problem considered here, the coupled pressure and temperature changes travel much slower than the uncoupled pressure disturbance. For example, at the observation point, indicated by an open circle in Figure 2, the coupled front arrives some time after 4,000 days (Figure 3) while the uncoupled pressure disturbance arrives at roughly 235 days (Figure 4). In order to compute the arrival time of the coupled front more accurately, I take the time derivatives of the temperature and pressure variations (Figure 7). In calculating the pressure derivative, some care must be exercised due to the presence of the earlier uncoupled pressure disturbance. That is, there is a very significant transient pressure response to the start of injection, independent of the thermal effects. In order to isolate the coupled pressure disturbance I consider the differential pressure, which is given by the pressure of the nonisothermal flow simulation, minus the pressure from an isothermal flow simulation. This differential pressure is plotted in Figure 7, with the pressure changes before the arrival of the thermal front set to zero. Note that the peak of the differential pressure coincides with the peak of the temperature derivative. The peak of the derivatives define the arrival times of the temperature and pressure changes.

As was done previously, I can compute the derivatives of the temperature and pressure with respect to time for each simulation grid block. In Figure $8 \mathrm{I}$ plot the normalized time derivatives for three 'snapshots' (801, 2125, and 4207 days). The temperature and pressure disturbances propagates outward, away from the injection well. However, the propagation speed is much slower for the coupled front, illustrating the importance of the thermal contributions to the slowness (74). The travel times for the temperature and pressure, which are related to the coupled phase $\theta(\mathbf{X})$, are plotted in Figure 9. As indicated in Figure 8, it takes several thousand days for the coupled front to propagate from the injection well to the observation well.

The asymptotic travel time estimates are obtained by solving the eikonal equation (73). In order to evaluate $\Omega$, given by (74), one needs estimates of $\bar{T}$ and $\bar{P}$ along the propagation paths of interest. These quantities were obtained from runs of the numerical simulator TOUGH2. In Figure 10 I have plotted the temperature and pressure variations for three observation points located 500, 1000, and 1500 meters from the injection well. The amplitude of the temperature change is relatively constant, roughly 135 degrees $\mathrm{C}$. The amplitude of the pressure change is the difference between the pressure following the pressure peak and the value of the pressure at the peak. The pressure change varies slowly with distance away from the injection well. In Figure 11 I have plotted the temperature and pressure changes over the simulation grid. From this figure it is clear that the temperature change is relatively constant and the pressure change is slowly-varying. These changes are used for $\bar{T}$ and $\bar{P}$ in the expression for $\Omega$ [equation (74)].
In an effort to examine the asymptotic travel times with the travel times estimated from the TOUGH2 simulation, I compare values along the trajectories extending from the injection well to the observation well. The trajectories, which for a homogeneous medium are straight lines, are plotted on the contour maps of the uncoupled and coupled travel times (Figures 6 and 9). The trajectories were obtained by solving the reduced ray equations, as described in Vasco and Finsterle [2004]. That is, I use the numerical simulator to compute the pressure and temperature time series in each grid block. By differentiating the time series and finding the time at which the derivative is a maximum I can estimate the distribution of the phase throughout the region of interest. I then use one of the ray equations to compute the trajectories by marching down the gradient of the phase function.

In the panel on the right-hand-side of Figure 12 I plot the travel times for both the pressure and the temperature variations for the case of coupled propagation. Due to the coupled nature of the propagation, the temperature and pressure travel times should lie close together. In general there is overall agreement between the temperature and pressure. The differences may be due to the approximate nature of the numerical calculations. For example, it was noted earlier that the propagating disturbances do not display the rotational symmetry of the problem due to grid effects. In addition, there are boundary effects to contend with, as well as rounding error and possible difficulties in integrating nonlinear differential equations. The asymptotic travel time estimates, as computed using the slowness given by equation (74), agree rather well with the temperature travel time.

\subsection{Propagation in a Heterogeneous Medium}

As mentioned above, the asymptotic approach, based upon the method of multiple scales [Anile et al., 1993, p. 49], is suited for modeling the propagation of disturbances in a medium with smoothly-varying heterogeneity. In an effort to illustrate this, I generated a heterogeneous distribution of permeability $(K)$, shown in Figure 13. Only the permeability was varied in this case, all other parameters were kept at the values used in the simulation in the homogeneous medium. In Figure 13 I have plotted the logarithm of permeability multipliers for each grid block, generated by forming the moving average of a distribution of random numbers. The radius of the averaging window controls the smoothness of the spatial distribution. The permeability varies by roughly two orders of magnitude about a mean value of $2 . \times 10^{-13} \mathrm{~m}^{2}$, with a low permeability region between the injection well and the observation well. The permeability is generally higher to the east of the injection well. The basic features of the numerical experiment, such as the location of the central injection and observation wells and the flow rates, are identical to the homogeneous example.

The procedures for a heterogeneous medium are identical to those used to model nonisothermal flow in a homogeneous medium. The numerical simulator TOUGH2 was used to solve the conservation of mass/momentum and energy. In order to capture the propagation of the pressure and temperature disturbances to the boundaries of the model domain, roughly 50,000 days of flow were simulated.

As in the homogeneous example, at the initiation of injection there is a pressure increase that propagates away from the central well. This propagation is clearly seen in Figure 14, where I have plotted the pressure change for three times (20, 204, and 692 days). The effect of the heterogeneity is seen in distribution of pressure change over time, as there is rapid propagation to the east, where the permeability is highest. The slowest propagation is to the northwest, 
through the low permeability zone between the injection well and the observation well. A contour plot of the travel time of the pressure disturbance, shown in Figure 15, confirms the faster propagation to the east and the slower propagation to the northwest. A trajectory, indicating the path of the transient disturbance from the injection well to the observation well, is shown by the curve in Figure 15 .

The temperature and pressure changes associated with the coupled propagation are shown in Figure 16. The coupled disturbance propagates at a much slower speed, indicating that the additional terms in the expression for slowness have a significant impact of the propagation. It takes more than 10,000 days for the disturbance to reach the observation well. The stands in contrast to the roughly 300 days required for the uncoupled pressure disturbance to propagate over the same distance. The temperature and pressure changes are similar in form and significantly different from the uncoupled pressure propagation in Figure 15, supporting the notion of coupled propagation. The propagation time through the layer is shown in Figure 17, along with the trajectory from the injection well to the observation well. The path curves in response to the heterogeneity, bending towards the region of higher permeability. This makes intuitive sense, as the first arriving disturbance should travel along the fastest path.

In Figure 18 I compare the travel times calculated from the output of the numerical simulator TOUGH2 (NUMERIC) to the asymptotic estimates, predicted by equation (74), for uncoupled and coupled propagation, respectively. As in the homogeneous medium, and as indicated in the snapshots of uncoupled and coupled propagation (Figures 14 and 16), the uncoupled pressure disturbance propagates much faster than the coupled disturbance. In spite of the fact that I used the expressions for a separable phase function, and did not account for the differences in temporal behavior of the amplitudes, there is generally good agreement between the travel times calculated using the asymptotic expression (74) and the values derived from the numerical simulation. Note that the heterogeneity, particularly the low permeabilities between the injection well and the observation well, changes the travel time to the observation point substantially. That is, it takes almost twice as long for the disturbances to propagated to the observation well.

\section{Conclusions}

It is possible to extend asymptotic techniques, developed for coupled linear systems of equations, to coupled nonlinear systems. In particular, using the method of multiple scales, an asymptotic approach, I have derived a semi-analytic solution for nonisothermal flow. Nonisothermal flow is governed by a set of partial differential equations containing coefficients that depend upon the temperature, rendering the problem nonlinear. Furthermore, due to the advection of heat there is a nonlinear term that is the scalar product of the pressure and thermal gradients. Thus, there are significant nonlinearities that become important when the temperature of an injected fluid differs substantially from that of the resident groundwater.

Assuming smoothly-varying heterogeneity, one can derive the condition that the governing equations have a nontrivial solution. Unlike the solution of the linear problem, this condition depends upon the amplitude jump due to the passage of the nonlinear disturbance. Therefore, the method requires a technique for estimating the amplitude of the pressure and temperature changes, such as a numerical simulator. Because of this, the technique is most useful in conjunction with a numerical reservoir simulator. The specific semi-analytic expressions provide insight into the medium parameters controlling the propagation of pressure and temperature changes in a heterogeneous porous medium. Furthermore, the technique provides explicit formulas for the travel time of the temperature and pressure in terms of the medium parameters, and may serve as the basis of an efficient inverse modeling scheme [Vasco and DattaGupta, 1999; Vasco et al., 2000; Datta-Gupta et al., 2002; Brauchler et al., 2003; Cheng and Datta-Gupta, 2005; He et al., 2006, Brauchler et al., 2007; Vasco, 2008; Brauchler et al., 2010]. The trajectories, or ray-paths, indicate those regions of the porous medium that influence the pressure and temperature disturbances observed at a particular location. As such, the trajectories and the expressions for the travel times may be used to compute semi-analytic sensitivities [Vasco and Datta-Gupta, 1999; Vasco et al., 2000; Vasco, 2008]. Inversions based upon transient arrival times are quasi-linear and thus less sensitive to the starting or prior model [Cheng et al., 2005]. Furthermore, arrival time inversions are relatively insensitive to boundary conditions and to flow properties outside the region of investigation.

The approach developed in this paper is applicable to more comprehensive treatments of coupled thermoelasticity and fluid flow [Brownell et al., 1977; Bear and Corapcioglu 1981; Noorishad et al., 1984]. As additional governing equations are added the expressions for the slownesses and travel times will become increasingly complicated. However, the formulas should provide valuable insight into these complex processes and aid in the interpretation of numerical simulations. The common formalism for coupled nonlinear flow and linear wave propagation provides some unity that might enhance our understanding. 


\section{Appendix A: The Method of Multiple Scales}

Asymptotic methods have a long history [Friedlander and Keller, 1955]. Asymptotic techniques have proven useful for a wide range of applications, from the modeling of electromagnetic [Kline and Kay, 1965] and seismic wave propagation to the modeling of fluid flow.

\subsection{The Conservation of Mass and Momentum}

In this sub-section I consider the first of the governing equations, equation (19), that I rewrite as

$$
\begin{aligned}
& \zeta_{P} \frac{\partial P}{\partial t}+\zeta_{T} \frac{\partial T}{\partial t}=\Upsilon_{P} \nabla \lambda \cdot \nabla P+\xi_{P T} \nabla T \cdot \nabla P \\
&+\Upsilon_{Z} \nabla \lambda \cdot \mathbf{Z}+\xi_{Z T} \nabla T \cdot \mathbf{Z} \\
&+\Upsilon_{P} \nabla \cdot \nabla P .
\end{aligned}
$$

I begin by substituting for the derivative operators in terms of differential operators with respect to the slow coordinates, as noted in the discussion surrounding equations (25), (26), and (27). The result is the extended expression

$$
\begin{gathered}
\zeta_{P}\left(\varepsilon \frac{\partial P}{\partial \tau}+\frac{\partial \theta_{P}}{\partial t} \frac{\partial P}{\partial \theta_{P}}\right) \\
+\zeta_{T}\left(\varepsilon \frac{\partial T}{\partial \tau}+\frac{\partial \theta_{T}}{\partial t} \frac{\partial T}{\partial \theta_{T}}\right)= \\
\Upsilon_{P} \varepsilon \nabla \lambda \cdot\left(\varepsilon \nabla P+\nabla \theta_{P} \frac{\partial P}{\partial \theta_{P}}\right) \\
+\xi_{P T}\left(\varepsilon \nabla T+\nabla \theta_{T} \frac{\partial T}{\partial \theta_{T}}\right) \cdot\left(\varepsilon \nabla P+\nabla \theta_{P} \frac{\partial P}{\partial \theta_{P}}\right) \\
+\Upsilon_{Z} \varepsilon \nabla \lambda \cdot \mathbf{Z} \\
+\xi_{Z T}\left(\varepsilon \nabla T+\nabla \theta_{T} \frac{\partial T}{\partial \theta_{T}}\right) \cdot \mathbf{Z} \\
+\Upsilon_{P} \varepsilon \nabla \cdot\left(\varepsilon \nabla P+\nabla \theta_{P} \frac{\partial P}{\partial \theta_{P}}\right) \\
+\Upsilon_{P} \nabla \theta_{P} \cdot \frac{\partial}{\partial \theta_{P}}\left(\varepsilon \nabla P+\nabla \theta_{P} \frac{\partial P}{\partial \theta_{P}}\right)
\end{gathered}
$$

where each term contains a factor that consists of $\varepsilon$ raised to some power. As noted in the Methodology section, $\varepsilon$ is the ratio of the length scale of the variation in pressure and temperature to the length scale of the heterogeneity. Because I am interested in modeling flow in a medium with smoothlyvarying heterogeneity, it is assumed that $\varepsilon$ is small, much less than one. Thus, I am primarily interested in terms of lowest order in $\varepsilon$. In particular, terms of order $\varepsilon^{0} \sim 1$ are retained in equation (A2), resulting in

$$
\begin{gathered}
\zeta_{P} \frac{\partial \theta_{P}}{\partial t} \frac{\partial P}{\partial \theta_{P}}+\zeta_{T} \frac{\partial \theta_{T}}{\partial t} \frac{\partial T}{\partial \theta_{T}}=\xi_{P T} \nabla \theta_{T} \cdot \nabla \theta_{P} \frac{\partial T}{\partial \theta_{T}} \frac{\partial P}{\partial \theta_{P}} \\
+\xi_{Z T} \nabla \theta_{T} \cdot \mathbf{Z} \frac{\partial T}{\partial \theta_{T}} \\
+\Upsilon_{P} \nabla \theta_{P} \cdot \nabla \theta_{P} \frac{\partial^{2} P}{\partial \theta_{P}^{2}} .
\end{gathered}
$$

As is done in wave propagation studies, I denote the gradient of the pressure phase function $\theta_{P}(\mathbf{X}, \tau)$ as a vector, the slowness vector $\mathbf{p}$,

$$
\mathbf{p}=\nabla \theta_{P} .
$$

Similarly, the gradient of the thermal phase function is represented by the vector

$$
\mathbf{s}=\nabla \theta_{T}
$$

which, in general, differs from p. Thus, I can rewrite equations (A3) as

$$
\begin{gathered}
\zeta_{P} \frac{\partial \theta_{P}}{\partial t} \frac{\partial P}{\partial \theta_{P}}+\zeta_{T} \frac{\partial \theta_{T}}{\partial t} \frac{\partial T}{\partial \theta_{T}}=\xi_{P T} \mathbf{s} \cdot \mathbf{p} \frac{\partial T}{\partial \theta_{T}} \frac{\partial P}{\partial \theta_{P}} \\
+\xi_{Z T} \mathbf{s} \cdot \mathbf{Z} \frac{\partial T}{\partial \theta_{T}} \\
+\Upsilon_{P} p^{2} \frac{\partial^{2} P}{\partial \theta_{P}^{2}}
\end{gathered}
$$

where $p$ and $s$ denote the magnitude of the vectors $\mathbf{p}$ and $\mathbf{s}$, respectively.

Now I can make use of the series representations (23) and (24) of $T\left(\mathbf{X}, \tau, \theta_{T}\right)$ and $P\left(\mathbf{X}, \tau, \theta_{T}\right)$, respectively, to evaluate the partial derivatives with respect to $\theta_{P}$ and $\theta_{T}$. Due to the specified form of the solutions, one can see that

$$
\frac{\partial T}{\partial \theta_{T}}=T-T_{b}=\bar{T}
$$

and

$$
\frac{\partial P}{\partial \theta_{P}}=P-P_{b}=\bar{P}
$$

where the bar signifies that the quantities represent the changes in temperature and pressure with respect to the background values $T_{b}(\mathbf{X}, \tau)$ and $P_{b}(\mathbf{X}, \tau)$. Note that, because $\varepsilon$ is assumed to be small, $\bar{P}$ is dominated by the change in pressure associated with the zeroth-order term, that is $\bar{P}=P_{0}-P_{b}$, similarly for $\bar{T}$. Using the expressions (A7) and (A8) for the partial derivatives of $P$ and $T$ with respect to the phase variable in equations (A6), and using (A9) to compute the derivative of the phase functions with respect to time, I arrive at

$$
\begin{gathered}
\zeta_{P} \bar{P} \frac{\partial \theta_{P}}{\partial t}+\zeta_{T} \bar{T} \frac{\partial \theta_{T}}{\partial t}-\xi_{P T} \mathbf{s} \cdot \mathbf{p} \bar{T} \bar{P}-\xi_{Z T} \mathbf{s} \cdot \mathbf{Z} \bar{T} \\
-\Upsilon_{P} p^{2} \bar{P}=0,
\end{gathered}
$$

a polynomial in the variables $\bar{P}$ and $\bar{T}$. Collecting the coefficients and rearranging terms, I can write equation (A9) as

$$
\xi_{P T} \mathbf{s} \cdot \mathbf{p} \bar{T} \bar{P}+\alpha_{P} \bar{P}-\alpha_{T} \bar{T}=0,
$$

where

$$
\alpha_{P}=\Upsilon_{P} p^{2}-\zeta_{P} \frac{\partial \theta_{P}}{\partial t}
$$

and

$$
\alpha_{T}=\zeta_{T} \frac{\partial \theta_{T}}{\partial t}+\xi_{Z T} \mathbf{s} \cdot \mathbf{Z}
$$

\subsection{The Conservation of Energy}

Now consider the second governing equation (19), expressing the conservation of energy,

$$
\begin{gathered}
\eta \frac{\partial T}{\partial t}=\nabla K_{T} \cdot \nabla T-C_{\rho} \Upsilon_{P} K \nabla P \cdot \nabla T-C_{\rho} \Upsilon_{Z} K \nabla T \cdot \mathbf{Z} \\
+K_{T} \nabla \cdot \nabla T .
\end{gathered}
$$

As in the previous sub-section, I can rewrite the differential operators in terms of the slow coordinates and the phase variables $\theta_{P}$ and $\theta_{T}$

$$
\begin{gathered}
\eta\left(\varepsilon \frac{\partial T}{\partial \tau}+\frac{\partial \theta_{T}}{\partial t} \frac{\partial T}{\partial \theta_{T}}\right)= \\
\varepsilon \nabla K_{T}\left(\varepsilon \nabla T+\nabla \theta_{T} \frac{\partial T}{\partial \theta_{T}}\right) \\
-C_{\rho} \Upsilon_{P} K\left(\varepsilon \nabla P+\nabla \theta_{P} \frac{\partial P}{\partial \theta_{P}}\right) \cdot\left(\varepsilon \nabla T+\nabla \theta_{T} \frac{\partial T}{\partial \theta_{T}}\right) \\
-C_{\rho} \Upsilon_{Z} K\left(\varepsilon \nabla T+\nabla \theta_{T} \frac{\partial T}{\partial \theta_{T}}\right) \cdot \mathbf{Z}
\end{gathered}
$$




$$
\begin{gathered}
+K_{T} \varepsilon \nabla \cdot\left(\varepsilon \nabla T+\nabla \theta_{T} \frac{\partial T}{\partial \theta_{T}}\right) \\
+K_{T} \nabla \theta_{T} \cdot \frac{\partial}{\partial \theta_{T}}\left(\varepsilon \nabla T+\nabla \theta_{T} \frac{\partial T}{\partial \theta_{T}}\right) .
\end{gathered}
$$

I consider terms of lowest order in $\varepsilon$, that is terms of order $\varepsilon^{0} \sim 1$, producing the expression

$$
\begin{gathered}
\eta \frac{\partial \theta_{T}}{\partial t} \frac{\partial T}{\partial \theta_{T}}=-C_{\rho} \Upsilon_{P} K \nabla \theta_{P} \cdot \nabla \theta_{T} \frac{\partial P}{\partial \theta_{P}} \frac{\partial T}{\partial \theta_{T}} \\
-C_{\rho} \Upsilon_{Z} K \nabla \theta_{T} \cdot \mathbf{z} \frac{\partial T}{\partial \theta_{T}}+K_{T} \nabla \theta_{T} \cdot \nabla \theta_{T} \frac{\partial^{2} T}{\partial \theta_{T}^{2}} .
\end{gathered}
$$

Making use of the definitions of the slowness vectors, (A4) and (A5), the expressions for the derivatives with respect to the phase variables, equations (A7) and (A8), I can write equation (A15) as

$$
\begin{array}{r}
C_{\rho} \Upsilon_{P} K \mathbf{p} \cdot \mathbf{s} \bar{T} \bar{P}-\eta \bar{T} \frac{\partial \theta_{T}}{\partial t}+C_{\rho} \Upsilon_{Z} K \mathbf{s} \cdot \mathbf{Z} \bar{T}-K_{T} s^{2} \bar{T}=0 . \\
(A 16)
\end{array}
$$

If I define the coefficient

$$
\beta_{T}=K_{T} s^{2}-C_{\rho} \Upsilon_{Z} K \mathbf{s} \cdot \mathbf{Z}+\eta \frac{\partial \theta_{T}}{\partial t}
$$

I can write equation (A16) as

$$
C_{\rho} \Upsilon_{P} K \mathbf{p} \cdot \mathbf{s} \bar{T} \bar{P}-\beta_{T} \bar{T}=0 .
$$




\section{Appendix B: Characteristics Separable Phase Function}

Though the numerical techniques described in the main body of this paper are the most general, additional insight is provided by an explicit solution for the phase function, the goal of this Appendix. Such a solution is possible if the equation governing the phase, equation (46) is separable. This will only be true if the gravitational term can be neglected and $\Omega$ is seperable. Therefore, I shall assume that gravitational forces are not important in the derivation that follows I shall also assume that the coefficient $\Omega$, given by equation (43), is only a function of spatial location $\mathbf{x}$ and is independent of time $t$. This will be true if the changes is pressure and temperature, $\bar{P}$ and $\bar{T}$, vary in a similar fashion as a function of time, say as $t^{-\alpha}$ for some constant $\alpha$, this will cancel in $\Omega$, as given by equation (43). Also, the time variation cancels when treating the propagation of uncoupled pressure or uncoupled temperature disturbances, the special cases considered above.

The simple structure of equation (46) when the gravity term vanishes, and the fact that the function $\Omega(\mathbf{x})$ only depends upon spatial position $\mathbf{x}$, might lead one to suspect that the separation of variables [Courant and Hilbert, 1962] could be used to find a solution. To this end, I assume that $\theta(\mathbf{x}, t)$ may be written in the form

$$
\theta(\mathbf{x}, t)=\beta(t) \sigma^{2}(\mathbf{x}),
$$

so that

$$
\nabla \theta=2 \beta \sigma \nabla \sigma
$$

and

$$
\frac{\partial \theta}{\partial t}=\sigma^{2} \frac{\partial \beta}{\partial t}
$$

Substituting these expressions into equation (46) results in

$$
4 \beta^{2} \nabla \sigma \cdot \nabla \sigma=\Omega \frac{\partial \beta}{\partial t}
$$

Because the terms in equation (B3) depend upon either $\mathbf{x}$ or $t$, but not both, I can group them as

$$
\frac{\nabla \sigma \cdot \nabla \sigma}{\Omega}=\frac{1}{4 \beta^{2}} \frac{\partial \beta}{\partial t}
$$

The left-hand-side of equation (B4) only depends upon $\mathbf{x}$, while the right-hand-side is only a function of $t$. Thus, each side must be equal to the same constant value, that I shall denote by $C$. Consider the right-hand-side, that can be written as an ordinary differential equation because $\beta$ only depends upon $t$,

$$
\frac{1}{4 \beta^{2}} \frac{d \beta}{d t}=C
$$

Equation (B5) can be integrated to produce an expression for $\beta(t)$

$$
\beta(t)=-\frac{1}{4 C t} .
$$

In all that follows I will take $C=1$, as this results in an expression for $\theta(\mathbf{x}, t)$ that resembles a solution of the linear diffusion equation.

Now I consider the left-hand-side of equation (B6), for $C=1$,

$$
\nabla \sigma \cdot \nabla \sigma=\Omega
$$

that I may write as

$$
F\left(\mathbf{x}, \mathbf{p}_{\sigma}\right)=\mathbf{p}_{\sigma} \cdot \mathbf{p}_{\sigma}-\Omega=0
$$

where I have defined the reduced slowness vector $\mathbf{p}_{\sigma}$ associated with $\sigma(\mathbf{x})$ :

$$
\mathbf{p}_{\sigma}=\nabla \sigma
$$

Equation (B7) is a first-order, nonlinear partial differential equation that can be solved by the method of characteristics. In fact, equation (B7) is the eikonal equation, a well known equation that appears in numerous wave propagation studies [Karal and Keller, 1959; Kline and Kay, 1965; Chapman, 2004]. By the method of characteristics, one can show that equation (B7) is equivalent to the system of ordinary differential equations [Courant and Hilbert, 1962, p. 97],

$$
\begin{gathered}
\frac{d x_{i}}{d s}=\frac{\partial F}{\partial p_{\sigma_{i}}}=p_{\sigma_{i}}, \\
\frac{d p_{\sigma_{i}}}{d s}=-\frac{\partial F}{\partial x_{i}}=\frac{1}{2} \frac{\partial \Omega}{\partial x_{i}} .
\end{gathered}
$$

Equations (B10) and (B11), written in vector form as

$$
\frac{d \mathbf{x}}{d s}=\mathbf{p}
$$

and

$$
\frac{d \mathbf{p}}{d s}=\frac{1}{2} \nabla \Omega
$$

comprise the ray equations that define the trajectory. From equation (B12) I have

$$
\mathbf{p}_{\sigma} \cdot \mathbf{p}_{\sigma}=p_{\sigma}{ }^{2}=\Omega
$$

which can be written in trajectory-based coordinates in which $\mathbf{p}_{\sigma}$ is tangent to the trajectory $\mathbf{x}(s)$. In trajectory based coordinates equation (B14) produces a differential equation for $\sigma$

$$
\frac{d \sigma}{d s}=\sqrt{\Omega}
$$

that can be integrated

$$
\sigma(\mathbf{x})=\int \sqrt{\Omega} d s .
$$

Thus, I can write the phase function as

$$
\theta(\mathbf{x}, t)=-\frac{\sigma^{2}(\mathbf{x})}{4 t}
$$

where $\sigma$ is given by (B17), a form that is similar to the phase associated with the diffusion equation in a heterogeneous medium [Vasco et al., 2000]. Equation (B17) provides a semi-analytic expression for the phase function. This expression can be combined with the definition of $\Omega$ in order to derive a representation of the phase function, $\theta$, entirely in terms of the parameters of the medium. 


\section{Appendix C: Analysis of the coefficient} $\Omega$

In this Appendix I examine the coefficient $\Omega$ that is present in the quadratic equation (44). The definition of $\Omega$, given in (43), is restated here for convenience:

$$
\Omega(\mathbf{x}, t, \theta)=\frac{\zeta_{P} \bar{P}+\left(\zeta_{T}+\eta \Delta\right) \bar{T}}{\Upsilon_{P} \bar{P}+K_{T} \Delta \bar{T}} .
$$

The coefficients $\zeta_{P}, \eta, \Delta, \Upsilon$, and $K_{T}$ depend upon the spatial coordinates and possibly upon the background pressures $\left(P_{b}\right)$ and temperatures $\left(T_{b}\right)$. The pressure and temperature changes, $\bar{P}$ and $\bar{T}$, are also present in expression (C1). It is though the presence of $\bar{T}$ and $\bar{P}$ that the phase variable $\theta$ appears in $\Omega(\mathbf{x}, t, \theta)$. For example, for a step-function source, the temperature and pressure disturbances are given to zeroth-order by the asymptotic power series (57) and (58). For an impulsive source-time function the zeroth-order temperature and pressure fields are given by

$$
\bar{T}(\mathbf{x}, \tau, \theta)=e^{\theta(\mathbf{x}, \tau)} T_{0}(\mathbf{x}, \tau)
$$

and

$$
\bar{P}(\mathbf{x}, \tau, \theta)=e^{\theta(\mathbf{x}, \tau)} P_{0}(\mathbf{x}, \tau),
$$

respectively, where $T_{0}(b f x, \tau)$ and $P_{0}(\mathbf{x}, \tau)$ are the amplitude functions. Here I shall briefly discuss the form of the coefficient $\Omega(\mathbf{x}, \tau, \theta)$ for both impulsive and step-function sources.

\subsection{An Impulsive Source-Time Function}

For an impulsive source, I can substitute the asymptotic solutions, given by (C2) and (C3), into the expression (C1). The $\exp (\theta)$ terms in the numerator and denominator cancel and one is left with

$$
\Omega(\mathbf{x}, t)=\frac{\zeta_{P} P_{0}(\mathbf{x}, \tau)+\left(\zeta_{T}+\eta \Delta\right) T_{0}(\mathbf{x}, \tau)}{\Upsilon_{P} P_{0}(\mathbf{x}, \tau)+K_{T} \Delta T_{0}(\mathbf{x}, \tau)} .
$$

Note that if the temperature and pressure amplitude functions $T_{0}(\mathbf{x}, \tau)$ and $P_{0}(\mathbf{x}, \tau)$ have the same time dependence, for example a power-law dependence with the same exponent, then $\Omega$ will only depend upon $\mathbf{x}$.

\subsection{A Step-Function Source}

For a step-function source, with a rapid onset followed by a constant flow rate, the zeroth-order asymptotic solutions are of the form (57) and (58). The amplitude and phase terms are coupled through the time integrals. However, the form of the integrand, a rapidly-varying exponential term multiplied by a more slowly-varying amplitude function, one can derive an asymptotic representation of the integral [Dingle, 1973, p. 110]. The approach is based upon a Taylor series expansion of the slowly-varying amplitude function and term by term integration. The resulting asymptotic expressions for the temperature and pressure variations are

$$
\bar{T}(\mathbf{x}, \tau, \theta)=\left(\alpha_{T}-2\right) ! e^{\theta(\mathbf{x}, \tau)} \tau^{-\alpha_{T}+2} \frac{\bar{T}_{0}(\mathbf{x})}{\left(\frac{\partial \theta}{\partial \tau}\right)^{\alpha_{T}-1}}
$$

and

$$
\bar{P}(\mathbf{x}, \tau, \theta)=\left(\alpha_{P}-2\right) ! e^{\theta(\mathbf{x}, \tau)} \tau^{-\alpha_{P}+2} \frac{\bar{P}_{0}(\mathbf{x})}{\left(\frac{\partial \theta}{\partial \tau}\right)^{\alpha_{P}-1}} .
$$

Upon the substitution of these expressions for $\bar{T}$ and $\bar{P}$ into equation (C1) for $\Omega(\mathbf{x}, t, \theta)$, the exponential phase terms cancel. However, the phase derivative terms, raised to the power of $\alpha_{T}-1$ and $\alpha_{P}-1$ do not appear to cancel unless the time dependencies are identical $\left(\alpha_{P}=\alpha_{T}\right)$.
Acknowledgments. This work was supported by the Assistant Secretary, Office of Basic Energy Sciences of the U. S. Department of Energy under contract DE-AC02-05CH11231. 


\section{References}

Anile, A. M., J. K. Hunter, P. Pantano, and G. Russo, Ray Methods for Nonlinear Waves in Fluids and Plasmas, Longman Scientific and Technical, New York, 1993.

Auriault, J. L., and P. Royer, Double conductivity media: A comparison between phenomenological and homogenization approaches, International Journal of Heat and Mass Transfer, 36, 2613-2621, 1993

Bear, J., and M. Y. Corapcioglu, A mathematical model for consolidation in a thermoelastic aquifer due to hot water injection or pumping, Water Resources Research, 17, 723-736, 1981.

Boberg, T. C., Thermal Methods of Oil Recovery, John Wiley and Sons, New York, 1988

Booker, J. R., and C. Savvidou, Consolidation around a spherical heat source, International Journal of Solids Structures, 20, 1079-1090, 1984

Bouche, D., F. Molinet and R. Mittra, Asymptotic Methods in Electromagnetics, Springer-Verlag, Berlin, 1997.

Brauchler, R., R. Liedl, and P. Dietrich, A travel time based hydraulic tomographic approach, Water Resources Research, 39, 1-12, 2003.

Brauchler, R., J. Cheng, M. Everett, B. Johnson, P. Dietrich, R. Liedl, and M. Sauter An integrated approach coupling hydraulic travel time tomography and amplitude inversion, Journal of Hydrology, doi:10.1016/j.jhydrol.2007.08.011, 2007.

Brauchler, R., R. Hu, T. Vogt, D. Halbourni, T. Heinrichs, T. Ptak, and M. Sauter Cross-well interference slug tests: An efficient tool for high resolution characterization of hydraulic heterogeneity, Journal of Hydrology, 384, 33-45, doi:10.1016/j.jhydrol.2010.01.004, 2010.

Brownell, D. H., S. K. Garg, and J. W. Pritchett, Governing equations for geothermal reservoirs, Water Resources Research, $\mathbf{1 3}$ 929-934, 1977.

Carslaw, H. S., and Jaeger, J. C., Conduction of Heat in Solids, Oxford University Press, Oxford, 1959

Cerveny, V., Seismic rays and ray intensities in inhomogeneous anisotropic media, Geophysical Journal of the Royal Astronomical Society, 29, 1-13, 1972.

Chapman, C. H., Fundamentals of Seismic Wave Propagation, Cambridge University Press, Cambridge, 2004.

Cheng, H., Z., He, and A. Datta-Gupta, A comparison of traveltime and amplitude matching for field-scale production data integration: Sensitivity, non-linearity, and practical implications, Soc. Petrol. Eng. Journal, 10, 75-90, 2005

Courant, R., and D. Hilbert, Methods of Mathematical Physics, Interscience, New York, 1962.

Cox, D., J. Little, and D. O'Shea, Using Algebraic Geometry, Springer, New York, 1998.

Crandall, M. G., and P.-L. Lions, Viscosity solutions of HamiltonJacobi equations, Transactions of the American Mathematical Society, 277, 1-43, 1983.

Crandall, M. G., L. C. Evans, and P.-L. Lions, Some properties of viscosity solutions of Hamilton-Jacobi equations, Transactions of the American Mathematical Society, 282, 487-502, 1984.

Crank, J., The Mathematics of Diffusion, Oxford University Press, London, 1975.

Datta-Gupta, A., S. Yoon, D. W. Vasco, and G. A. Pope, Inverse modeling of partitioning interwell tracer tests: A streamline approach, Water Resources Research, 38, 1-15, doi:10.1029/2001WR000597,2002

de Marsily, G., Quantitative Hydrogeology, Academic Press, San Diego, 1986.

Dingle, R. B., Asymptotic Expansions: Their Derivation and Interpretation, Academic Press, 1973.

Doughty, C., and K. Pruess, A similarity solution for two-phase water, air, and heat flow near a linear heat source in a porous medium, Journal of Geophysical Research, 97, 1821-1838, 1992

Eaton, D. W. S., Finite difference travel-time calculation for anisotropic media, Geophysical Journal International, 114, 273-280, 1993.

Friedlander, F. G., and J. B. Keller. Asymptotic expansions of solutions of $\left(\nabla^{2}+k^{2}\right) u=0$, Communications of Pure and Applied Mathematics, 8, 387, 1955.

Grifoll, J., J. M. Gasto, and Y. Cohen, Non-isothermal soil water transport and evaporation, Advances in Water Resources, 28, $1254-1266,2005$
He, Z., A. Datta-Gupta, and D. W. Vasco, Rapid inverse modeling of pressure interference tests using trajectory-based traveltime and amplitude sensitivities, Water Resources Research, 42, W03419, doi:10.1029/2004/WR003783.

Hsiao, K.-T., and S. G. Advani, A theory to describe heat transfer during laminar incompressible flow of a fluid in periodic porous media, Physics of Fluids, 11, 1738-1748, 1999.

Iyer, H. M., and K. Hirahara, Seismic Tomography: Theory and Practice, Chapman and Hall, London, 1993.

Karal, F. C., and J. B. Keller, Elastic wave propagation in homogeneous and inhomogeneous media, Journal of the Acoustical Society of America, 31, 694-705, 1959.

Kline, M., and I. W. Kay, Electromagnetic Theory and Geometrical Optics, John Wiley and Sons, New York, 1965.

Kravtsov, Y. A., and Y. I. Orlov, Geometrical Optics of Inhomogeneous Media, Springer-Verlag, Berlin, 1990.

Lanczos, C., The Variational Principles of Mechanics, Dover Publications, New York, 1986.

Lecomte, I., Finite difference calculation of first traveltimes in anisotropic media, Geophysical Journal International, $\mathbf{1 1 3}$ 318-342, 1993

LeVeque, R. J., Numerical Methods for Conservation Laws, Birkhauser, Basel, 1992.

Maslov, V. P., and G. A. Omel'yanov, Geometric Asymptotics for Nonlinear PDE. I, American Mathematical Society, Providence, Rhode Island, 2001.

McTigue, D. F., Thermoelastic response of fluid-saturated porous rock, Water Resources Research, 91, 9533-9542, 1986.

Mercer, J. W., and C. R. Faust, A review of numerical simulation of hydrothermal systems, Hydrological Sciences Bulletin, 24, 335-343, 1979

Moyne, C., Two-equation model for a diffusive process in porous media using the volume averaging method with an unsteadystate closure, Advances in Water Resources, 20, 63-76, 1997.

Natale, G., and E. Salusti, Transient solutions for temperature and pressure waves in fluid-saturated porous rocks, Geophysical Journal International, 124, 649-656, 1996.

Noorishad, J., C. F. Tsang, and P. A. Witherspoon, Coupled thermal-hydraulic-Mechanical phenomena in saturated fractured porous rocks: Numerical approach, Water Resources Research, 89, 10365-10373, 1984

O'Sullivan, M. J., A similarity method for geothermal well test analysis, Water Resources Research, 17, 390-398, 1981.

Pinder, G., F., State-of-the-Art Review of Geothermal Reservoir Modelling, LBNL Report, 9093, Berkeley, 1979.

Pruess, K., C. Oldenburg, and G. Moridis, TOUGH2 User's Guide, Version 2.0, LBNL Report, 43134, Berkeley, 1999.

Qian, J., and W. W. Symes, Paraxial eikonal solvers for anisotropic quasi-P travel times, Journal of Computational Physics, 173, 256-278, 2001.

Quintard, M., M. Kaviany, and S. Whitaker, Two-medium treatment of heat transfer in porous media: numerical results for effective properties, Advances in Water Resources, 20, 77-94, 1997.

Ruter, A., Aspects of modern raytracing application design, Studia Geophysica et Geodaetica, 48, 143-165, 2004.

Sethian, J. A., Numerical algorithms for propagating interfaces: Hamilton-Jacobi equations and conservation laws, Journal of Differential Geometry, 31, 131-161, 1990.

Sethian, J. A., Level Set Methods, Cambridge University Press, 1999.

Sethian, J. A., and J. D. Strain, Crystal growth and dendritic solidification, Journal of Computational Physics, 98, 231-253, 1992.

Soukina, S. M., D. Gajewski, and B. M. Kashtan, Traveltime computation for 3D anisotropic media by a finite-difference perturbation method, Geophysical Prospecting, 51, 431-441, 2003.

Sozer, E., and W. Shyy, Multi-scale thermo-fluid transport in porous media, International Journal of Numerical Methods for Heat and Fluid Flow, 18, 883-899, 2008.

van Trier, J., and W. W. Symes, Upwind finite-difference calculations of traveltimes, Geophysics, 56, 812-821, 1991.

Vasco, D. W., An asymptotic solution for two-phase flow in the presence of capillary forces, Water Resources Research, 40 , W12407, 2004.

Vasco, D. W., Zeroth-order inversion of transient pressure observations, Inverse Problems, 24, 1-21, 2008. 
Vasco, D. W., Modelling broad-band poroelastic propagation using an asymptotic approach, Geophysical Journal International, 179, 299-318, 2009

Vasco, D. W., and A. Datta-Gupta, Asymptotic solutions for solute transport: A formalism for tracer tomography, Water Resources Research, 35, 1-16, 1999.

Vasco, D. W., and Finsterle, S., Numerical trajectory calculations for the efficient inversion of flow and tracer observations, Water Resources Research, 40, W01507, 1-17, 2004.

Vasco, D. W., H. Keers, and K. Karasaki, Estimation of reservoir properties using transient pressure data: An asymptotic approach, Water Resources Research, 36, 3447-3465, 2000.
Vasco, D. W., A. Ferretti, and F. Novali, Estimating permeability from quasi-static deformation: Temporal variations and arrival-time inversion, Geophysics, 73, O37-O52, 2008.

Virieux, J., C. Flores-Luna, and D. Gibert, Asymptotic theory for diffusive electromagnetic imaging, Geophysical Journal International, 119, 857-868, 1994.

D. W. Vasco, Earth Sciences Division/Building 90, Berkeley Laboratory, 1 Cyclotron Road, Berkeley, CA 94720. (email:dwvasco@lbl.gov) 
Figure 1. The variation of the fluid density (Upper panel) and viscosity (Lower panel) as a function of the fluid pressure and temperature. The range of the pressure and temperature were chosen to reflect the variations present in the numerical simulations.

Figure 2. The pressure and temperature distribution 4,207 days after the initiation of injection. High temperature water, with an enthalpy of $7.0 \times 10^{5} \mathrm{~J} / \mathrm{kg}$, is injected into the central well (denoted by an unfilled star) at a rate of $10.0 \mathrm{~kg} / \mathrm{s}$. The observation location, located in the upper-left-hand-corner of each of the two panels, is indicated by an open circle.

Figure 3. Pressure and temperature variations calculated for the observation location in Figure 2 (open circle). The pressures for both isothermal and nonisothermal simulations are shown.

Figure 4. A more detailed view of the pressure variation over the first 500 days of injection. Both isothermal and nonisothermal simulations are shown for comparison. The time derivative of the pressure variations are shown in the panel on the right.

Figure 5. Three snapshots of the time derivative of the pressure variation, normalized by the peak value in each grid block. The figures correspond to the uncoupled pressure variation, the early-time pressure variation, as shown in Figure 4.

Figure 6. The travel time associated with the propagation of the uncoupled pressure disturbance. The arrival time of the pressure disturbance corresponds to the time at which the time derivative of the pressure is a maximum. The line connecting the injection well (star) and the observation well (open circle) is the trajectory, signifying the travel path of the uncoupled pressure disturbance.

Figure 7. The time derivative of the temperature and pressure variations, as calculated by the TOUGH2 reservoir simulator [Pruess et al., 1999].

Figure 8. (Left Panel) Three snapshots of the normalized time derivative of the coupled temperature variation in the simulation grid.

(Right Panel) Three snapshots of the normalized time derivative of the coupled pressure variation in the simulation grid.

Figure 9. (Left Panel) The travel time of the coupled temperature disturbance. The travel time corresponds to the time at which the temperature derivative is a peak for each grid block of the simulation grid. The line connecting the injection well to the observation location is the ray-path, denoting the propagation path of the temperature disturbance.

(Right Panel) The travel time of the coupled pressure disturbance. The travel time corresponds to the time at which the pressure derivative is a peak for each grid block of the simulation grid. The line connecting the injection well to the observation location is the propagation path of the coupled pressure disturbance. 
Figure 10. (A.) Temperature change from the background value corresponding to three observation points located at 500, 1000, and 1000 meters from the injection well.

(B.) The pressure change corresponding to the coupled pressure and temperature disturbance. Each curve represents the variation of pressure with time, as calculated by the numerical simulation code TOUGH2. The calculations are for observation points 500, 1000, and $1500 \mathrm{~m}$ from the injection well.

Figure 11. (Left Panel) Temperature amplitude changes from the initial background values. (Right Panel) Pressure amplitude changes from the background values.

Figure 12. The travel times for various points along the trajectories in Figures 6 and 9. Travel times were obtained by post-processing the output of the TOUGH2 numerical simulator [NUMERIC]. (Left Panel) These travel time estimates are associated with uncoupled pressure propagation. The values output from the numerical simulator TOUGH2 [NUMERIC] were compared with calculations made using the asymptotic expressions for the travel times, from equations (71) and (72) [ASYMPTOTIC]

(Right Panel) For coupled propagation, both the pressure [PRESSURE] and temperature [TEMPERATURE] arrival times are plotted with the asymptotic estimates [ASYMPTOTIC]. The asymptotic estimates were obtained by solving equation (73) where $\Omega$ was given by equation (74).

Figure 13. Permeability variation used in the heterogeneous simulation. The darker colors indicate higher permeability regions while the lighter yellows indicate low permeability regions. As in the previous example, the injection well is indicated by the unfilled star in the center of the grid. The observation point is denoted by the unfilled circle.

Figure 14. Three snapshots of the normalized time derivative of pressure. The pressure variation corresponds to uncoupled pressure propagation. 
Figure 15. The travel time associated with the propagation of the uncoupled pressure disturbance in the heterogeneous medium shown in Figure 13. The curve connecting the injection well (star) and the observation well (open circle) is the trajectory, signifying the travel path of the uncoupled pressure disturbance.

Figure 16. Snapshots of the temperature and pressure derivatives associated with the coupled propagation in the heterogeneous medium.

Figure 17. The travel time associated with the propagation of the coupled pressure and temperature disturbance in the heterogeneous medium. The curve connecting the injection well and the observation well signifies the travel path of the coupled disturbance.

Figure 18. The travel times for various points along the trajectories in Figures 15 and 17. Travel times were obtained by post-processing the output of the TOUGH2 numerical simulator [NUMERIC]. (Left Panel) Uncoupled pressure propagation.

(Right Panel) Coupled pressure and temperature propagation. The estimates from the TOUGH2 simulation were compared with calculations made using the asymptotic expressions for the travel times, from equations (73) and (74) [ASYMPTOTIC], where the permeability now varies along the trajectory. 


\section{Density}

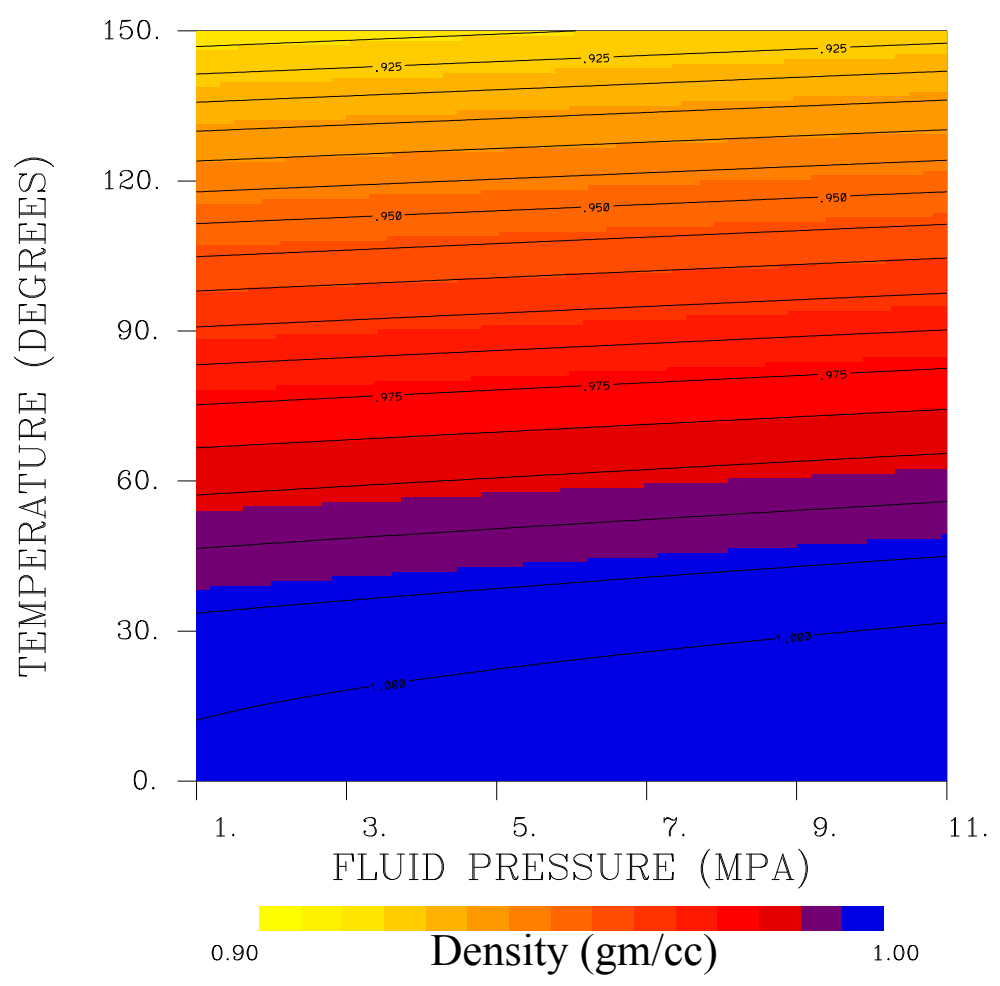

\section{Viscosity}

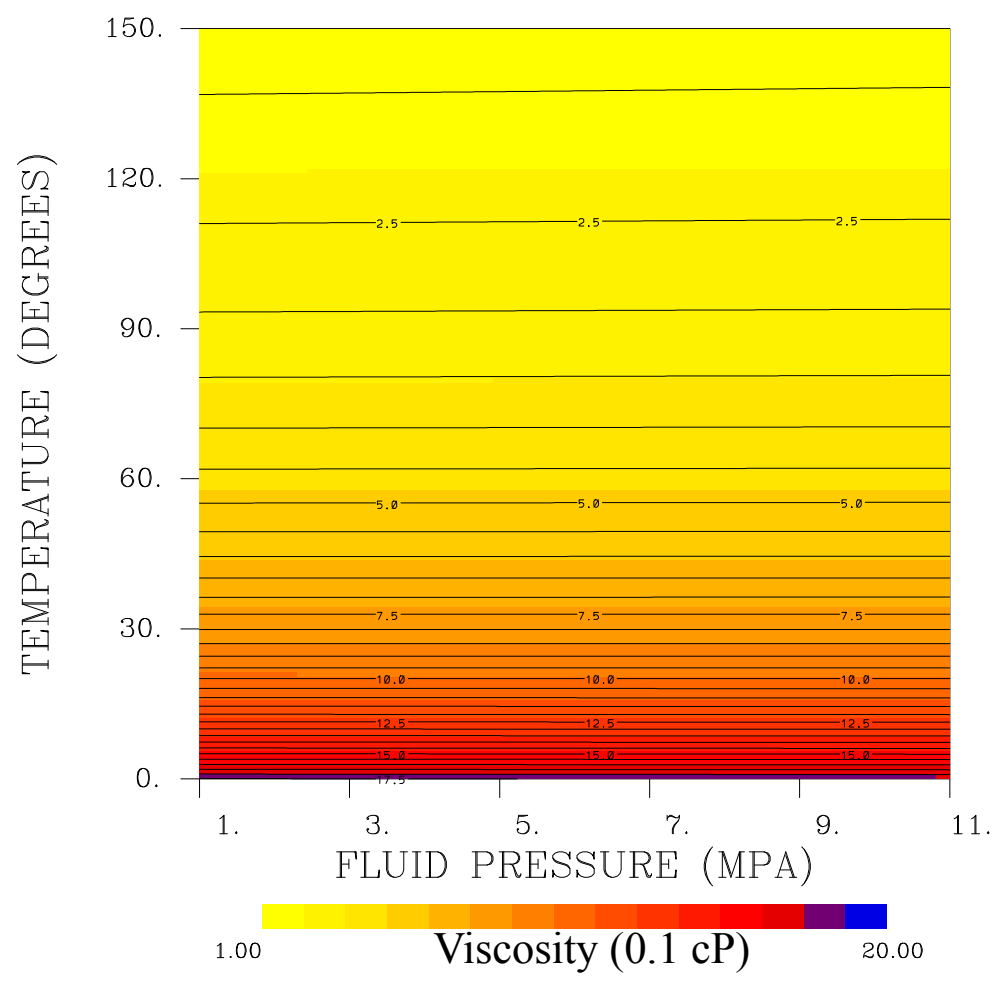

Eigure 1. 

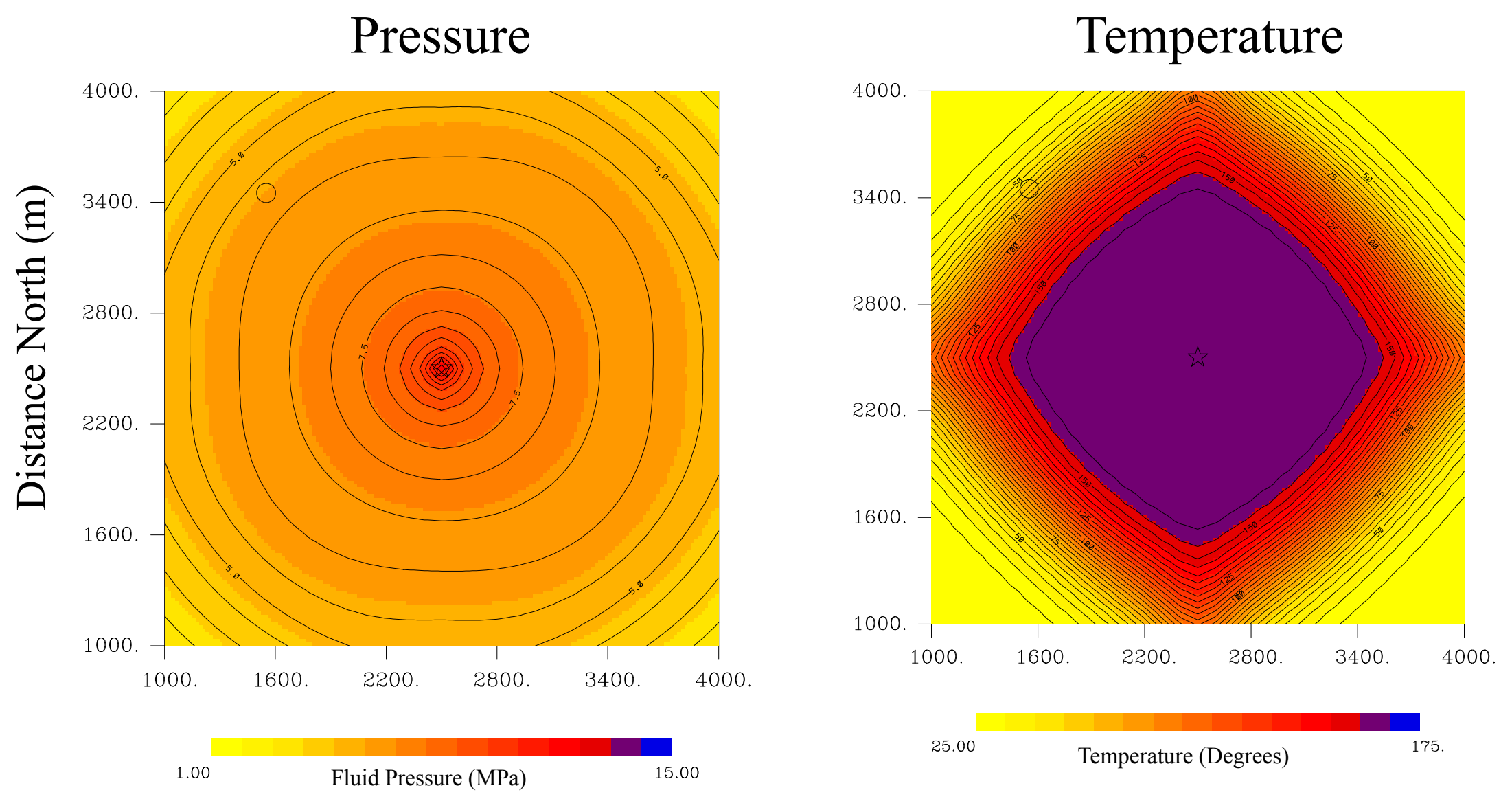

Figure 2 . 


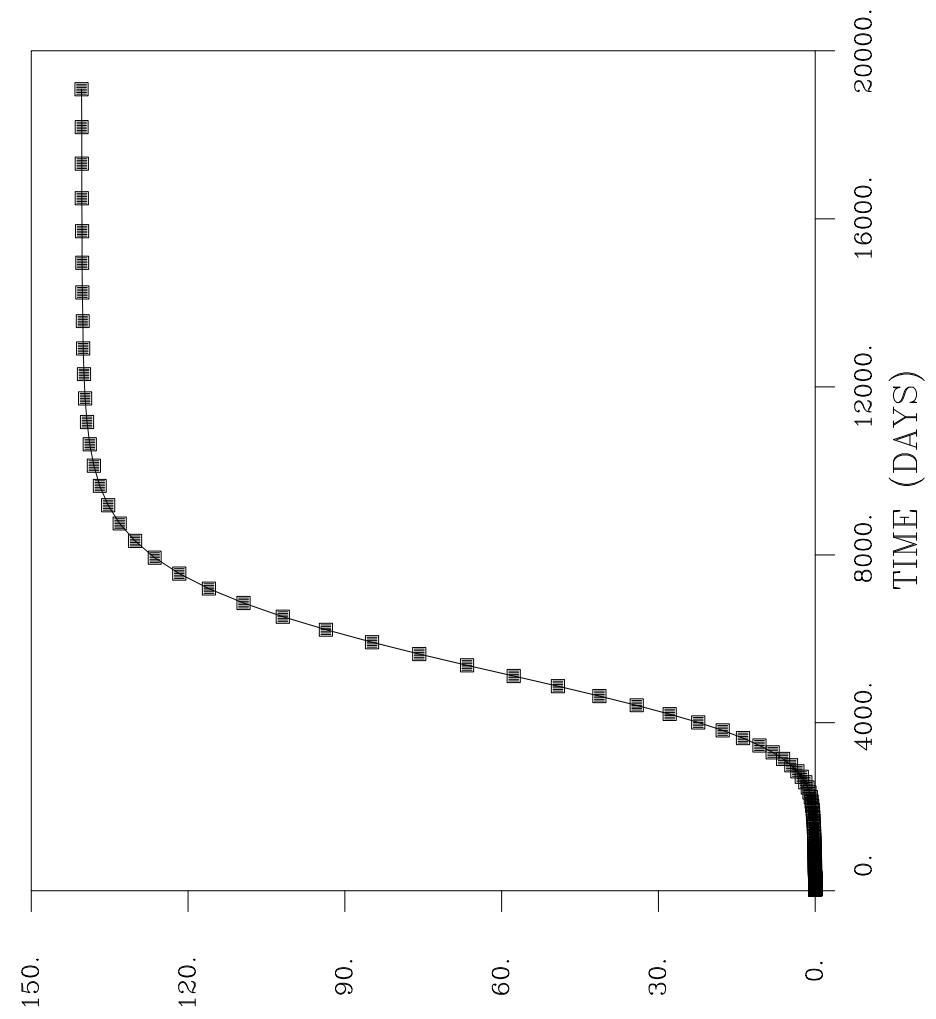

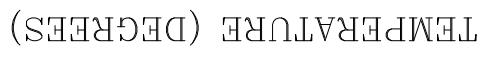

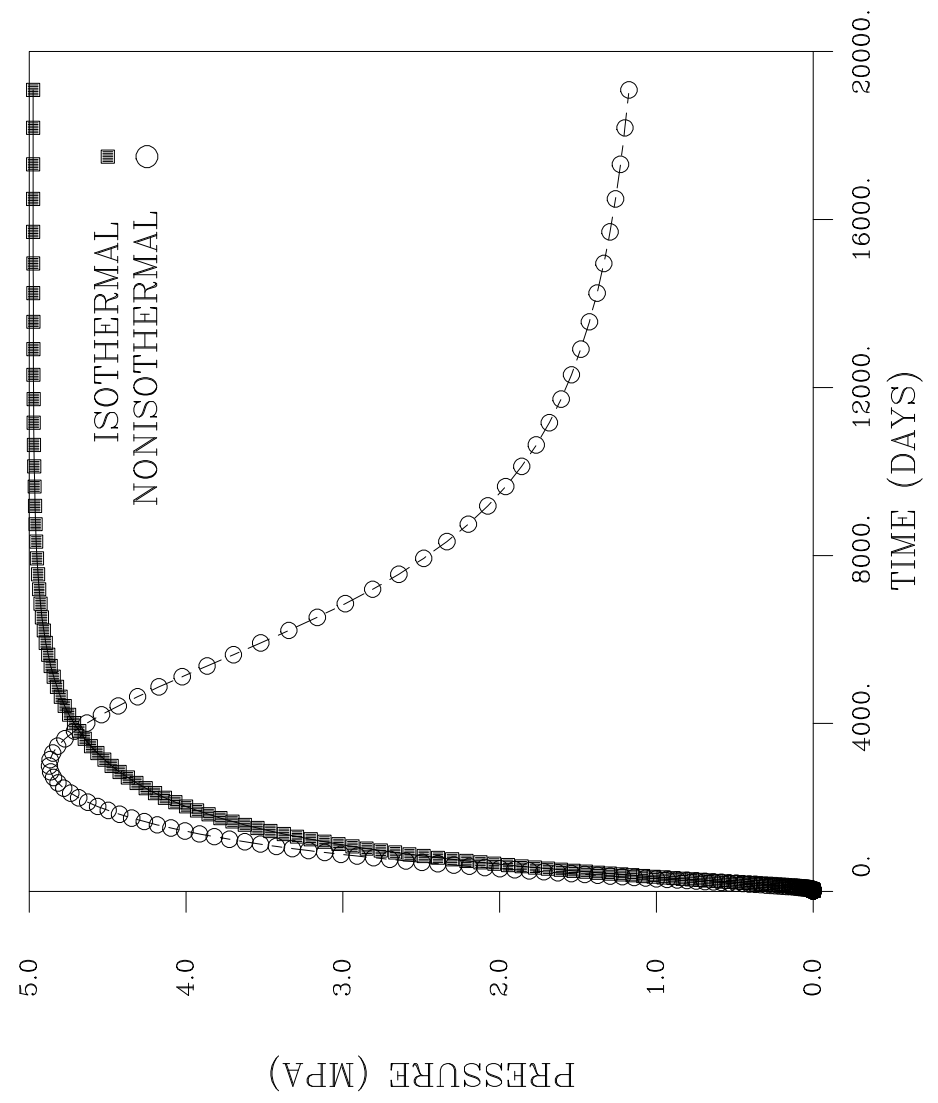

Figure 3. 

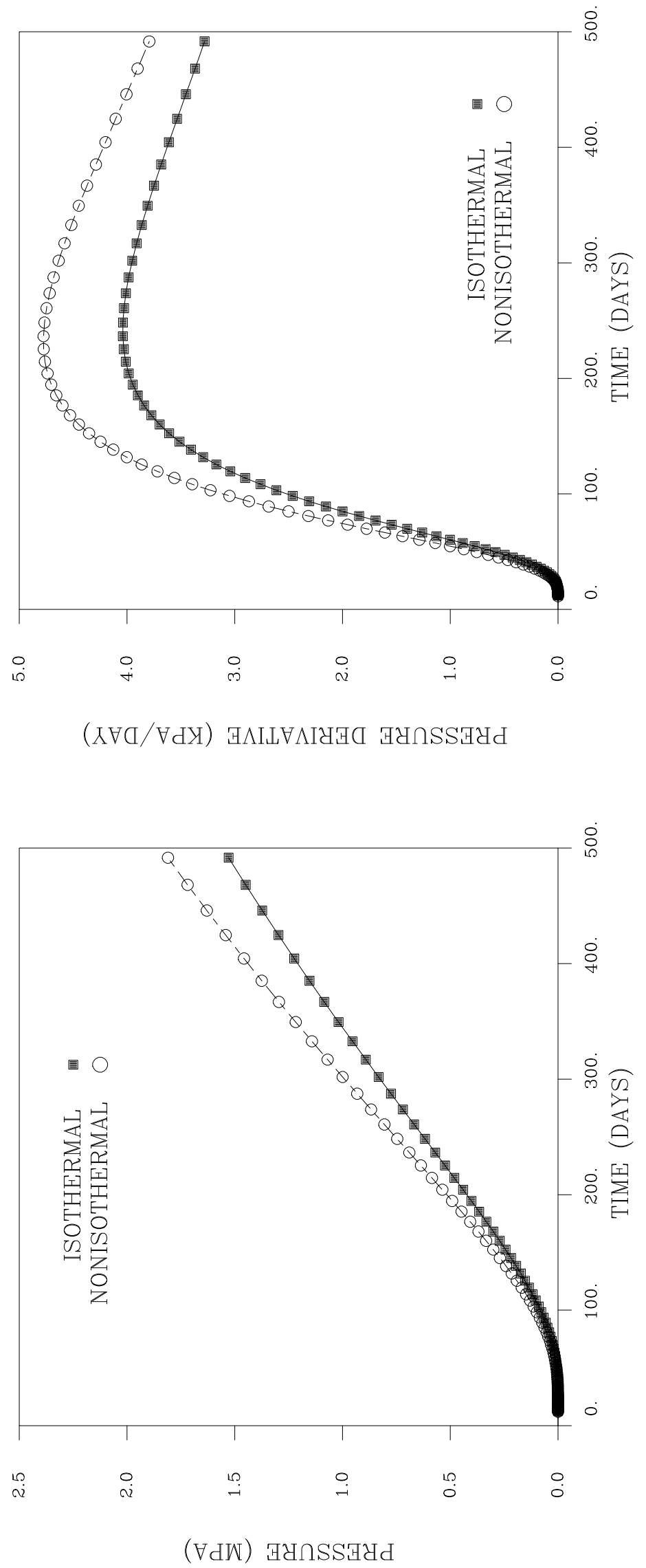

Figure 4. 


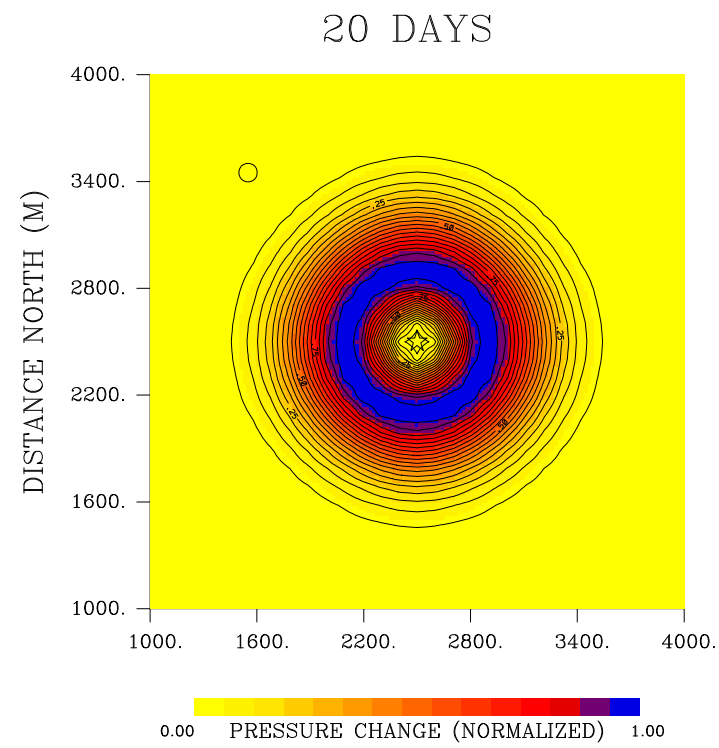

98 DAYS

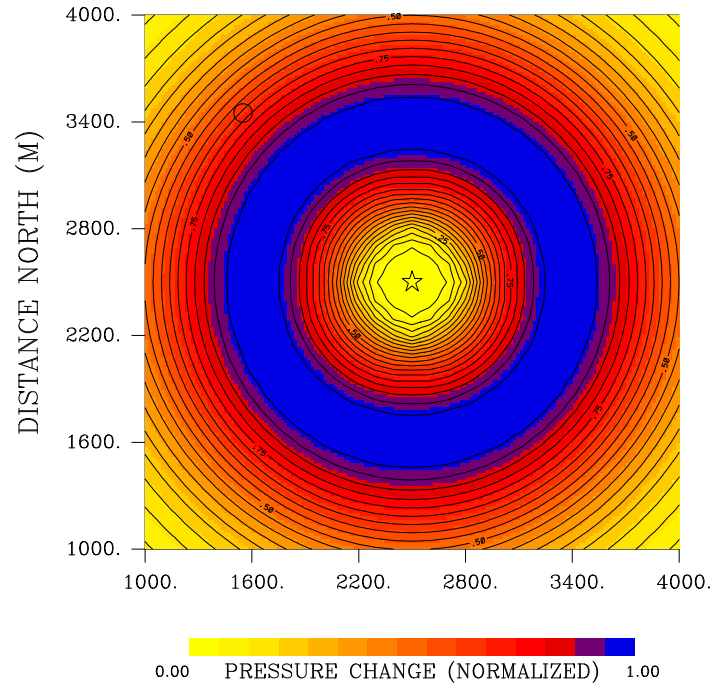

446 DAYS

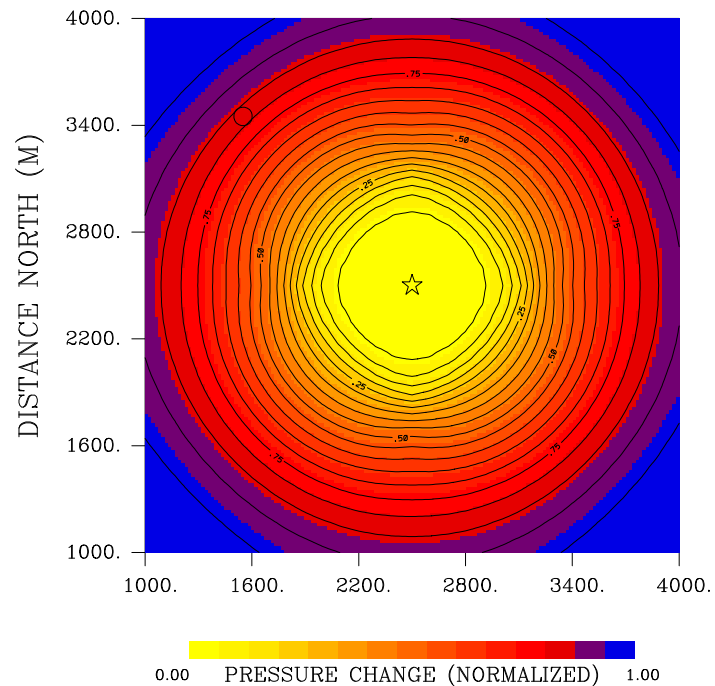




\section{Uncoupled Pressure}

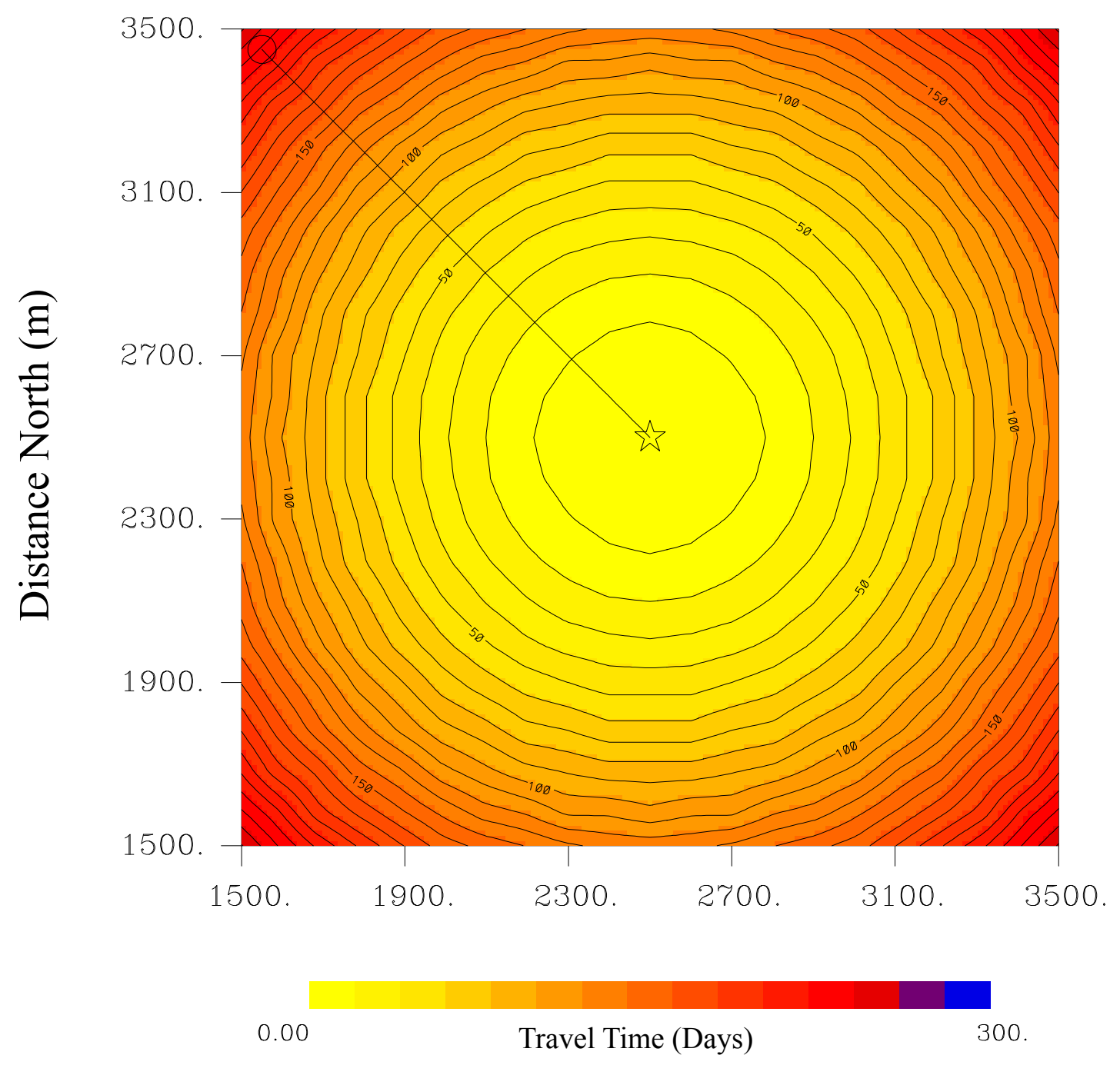

Figure 6. 


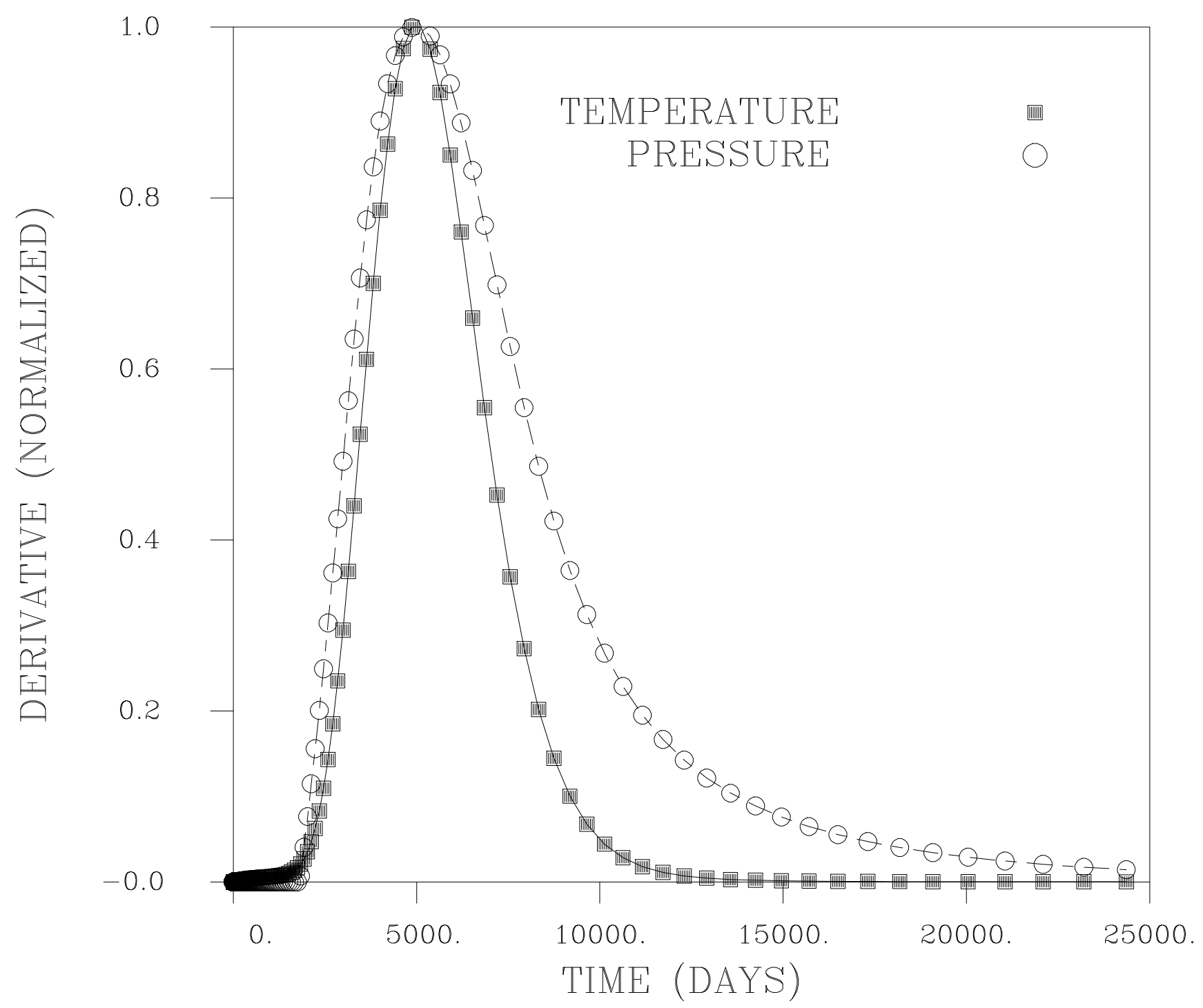

Figure 7 . 


\section{Temperature}

801 DAYS

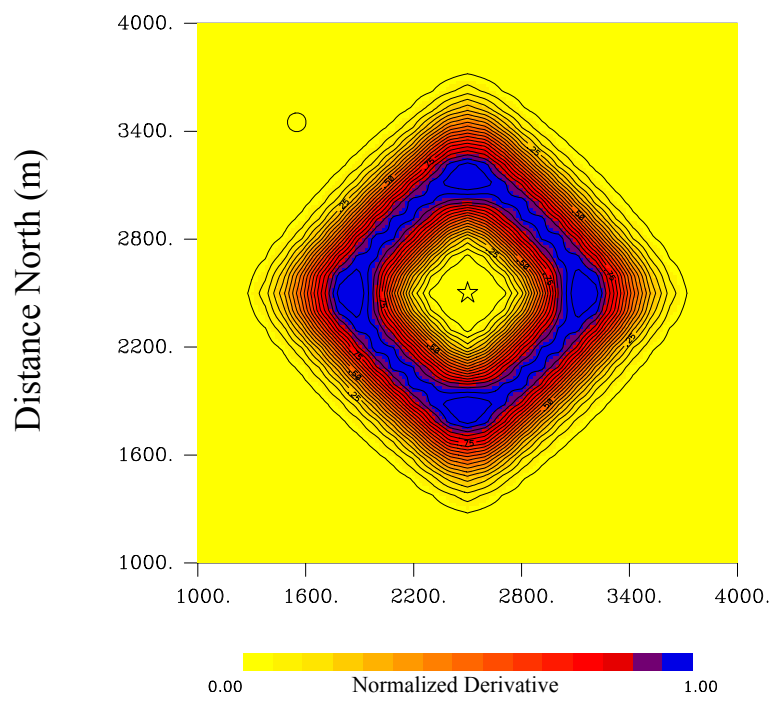

2125 DAYS

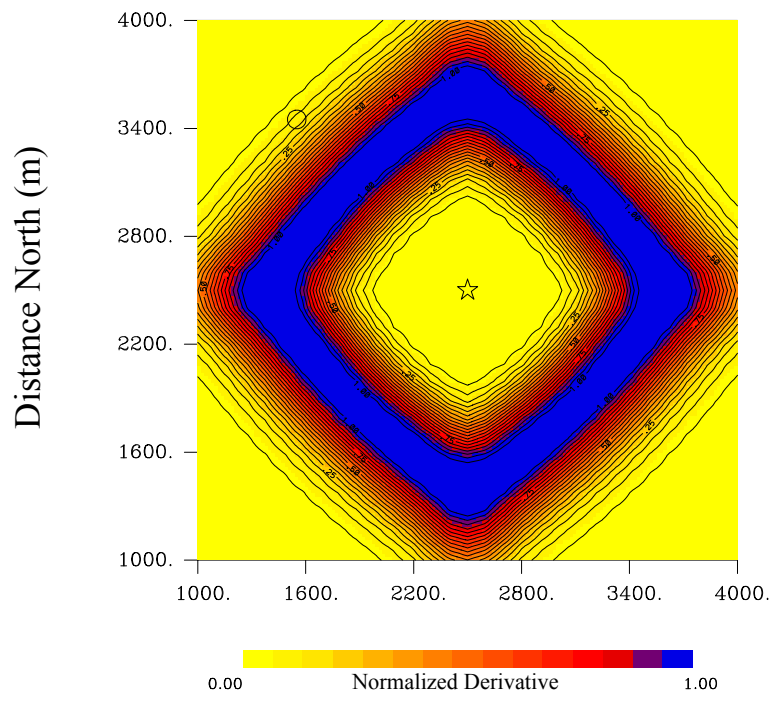

4207 DAYS

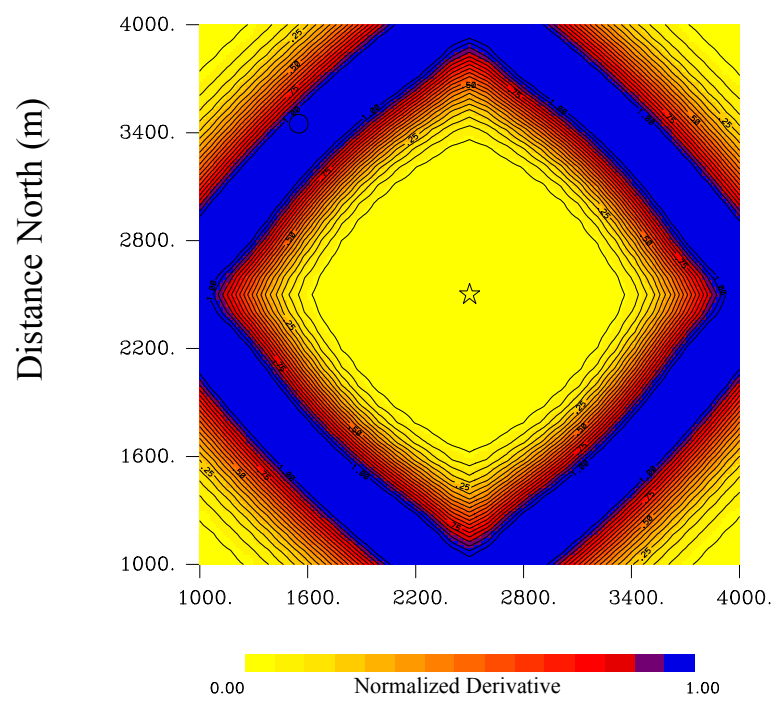

Pressure
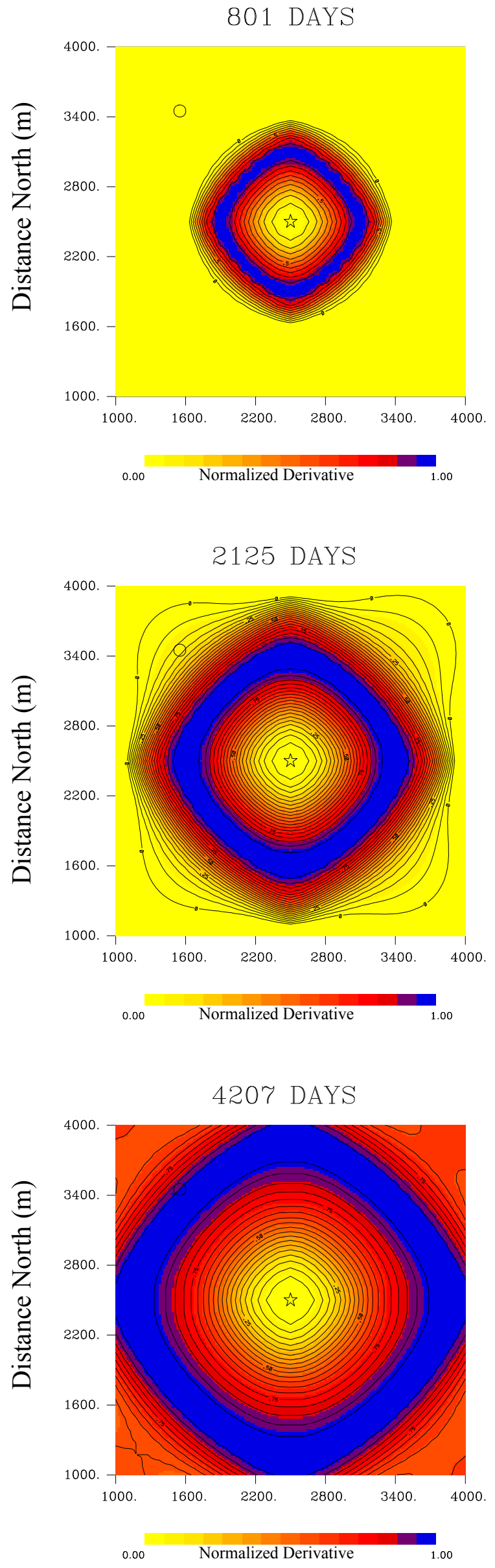


\section{Temperature}

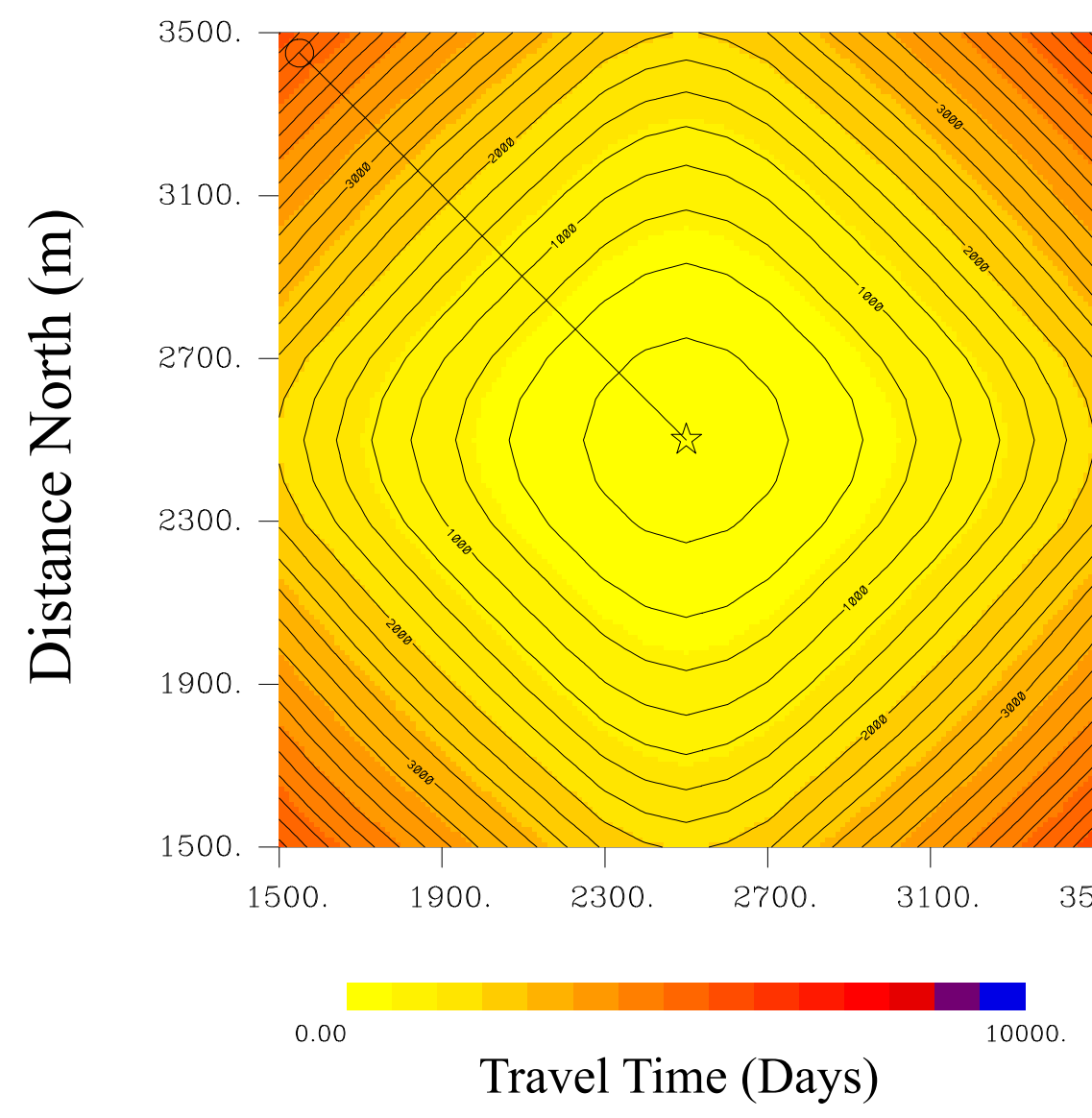

Pressure
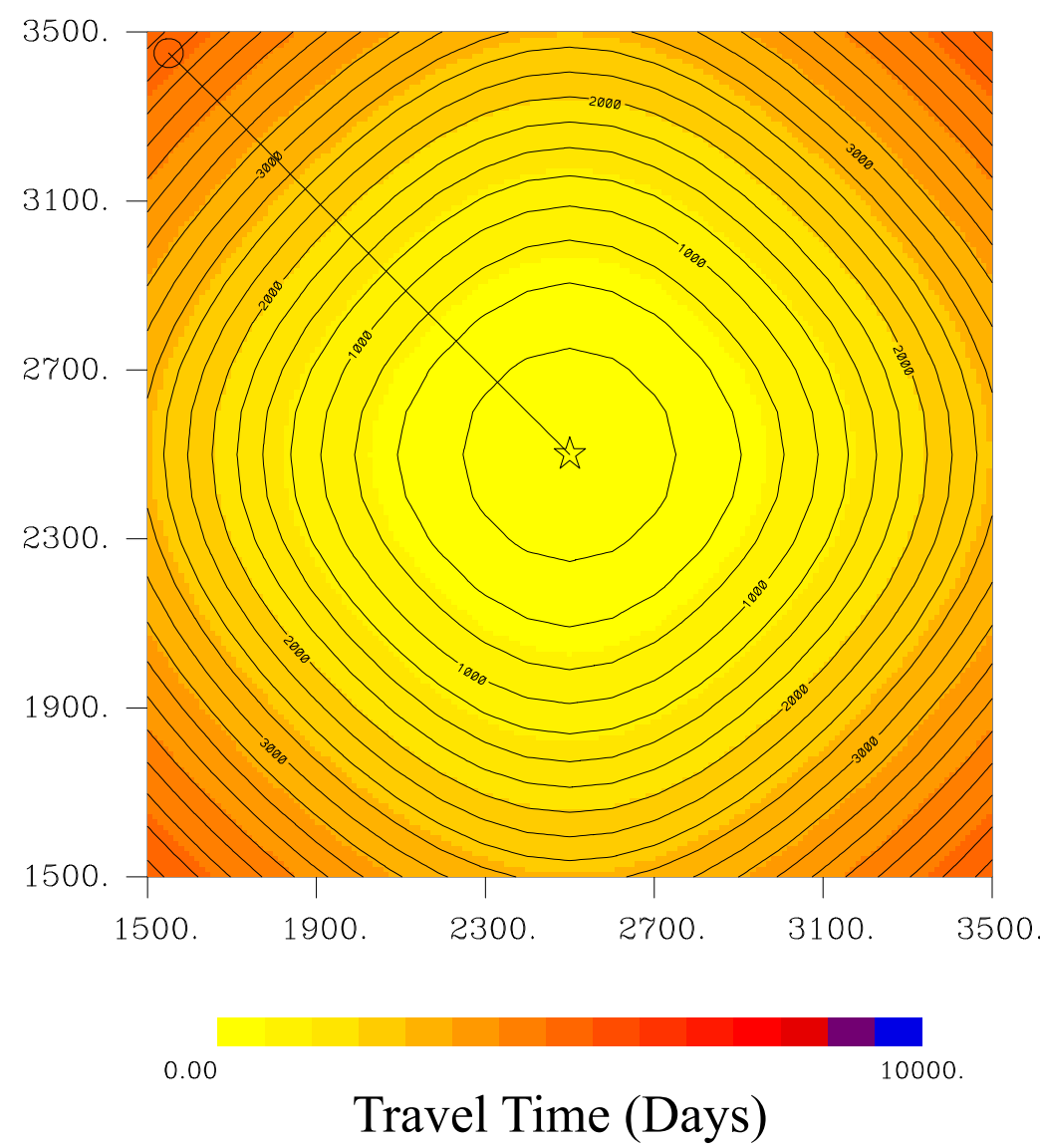


\section{Temperature}

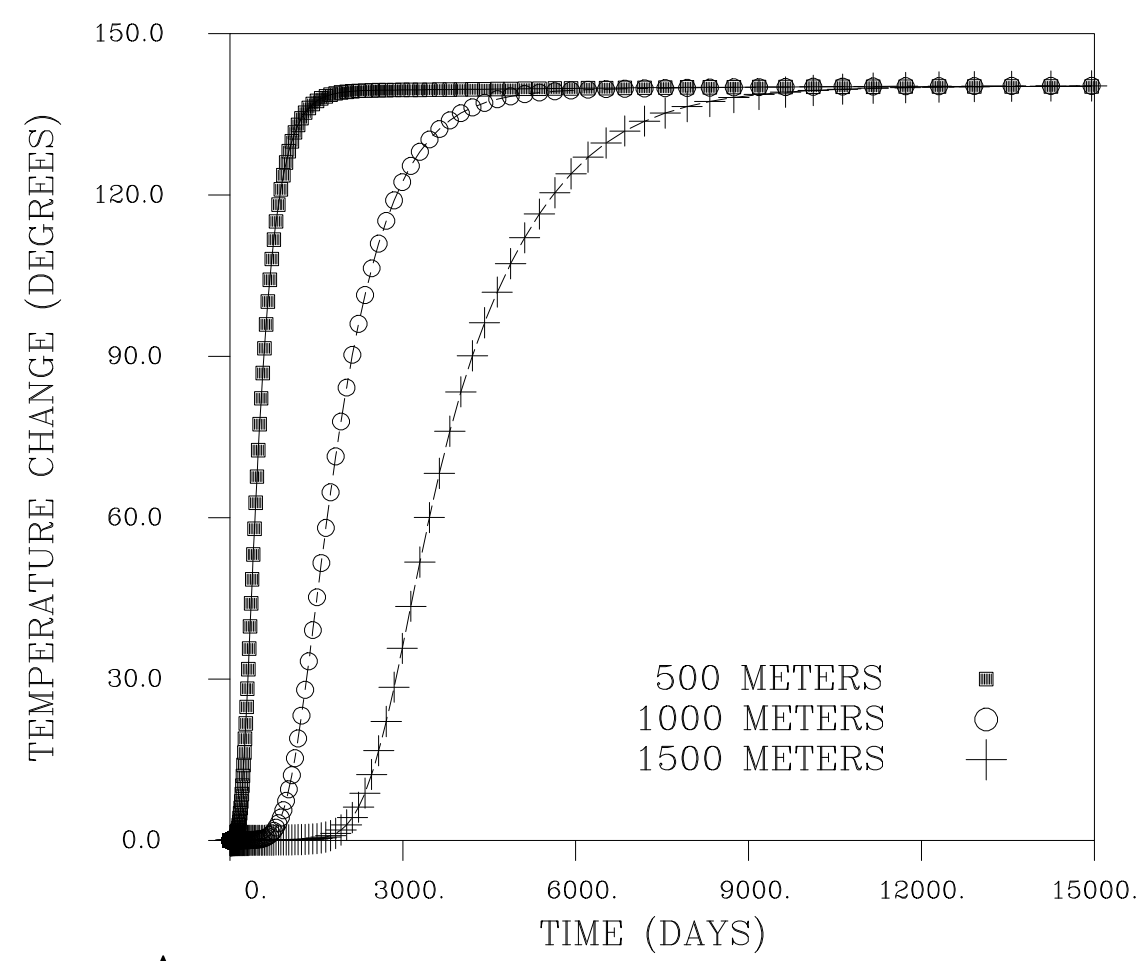

A.

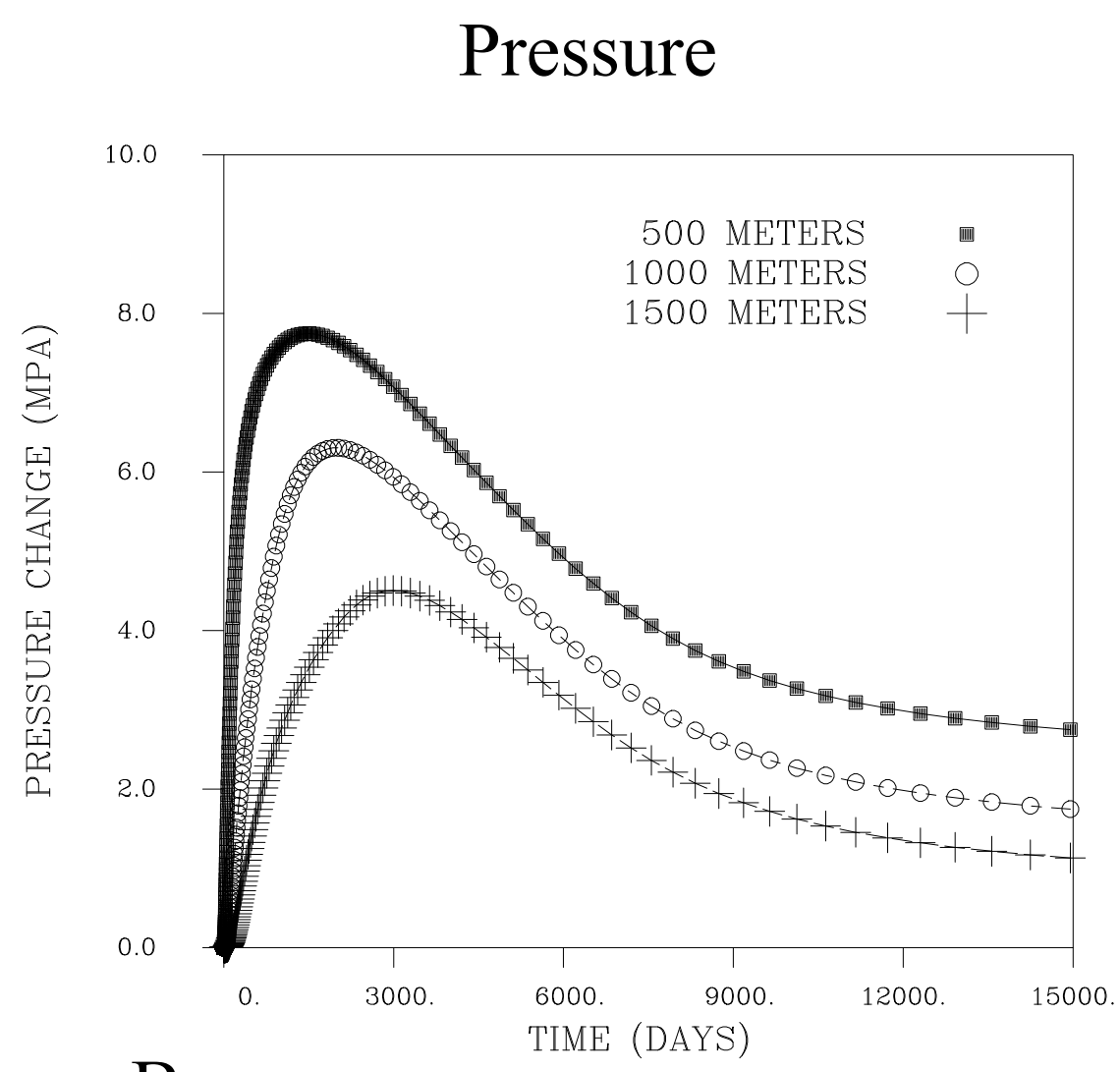

B. 
Temperature

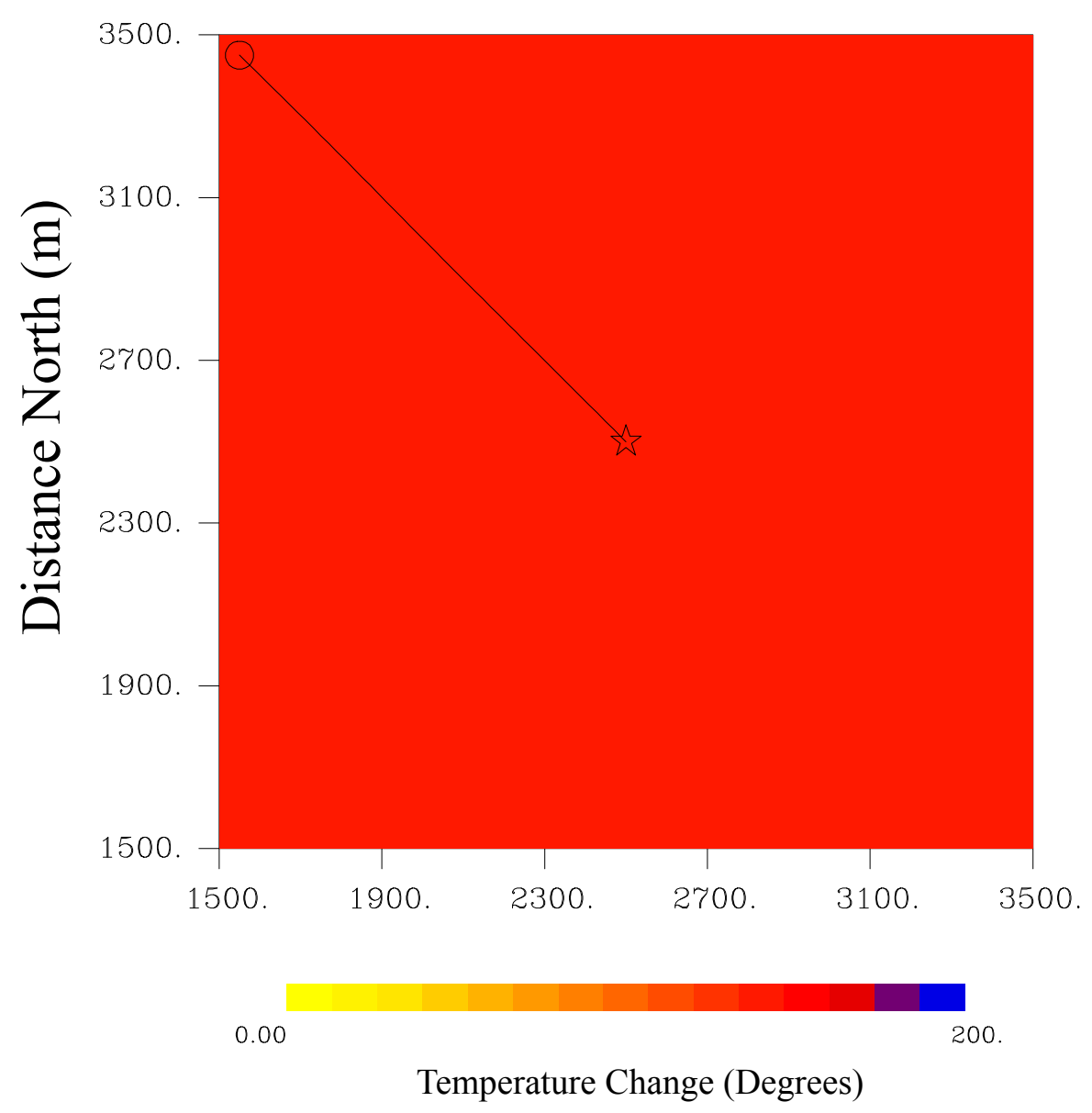

Pressure

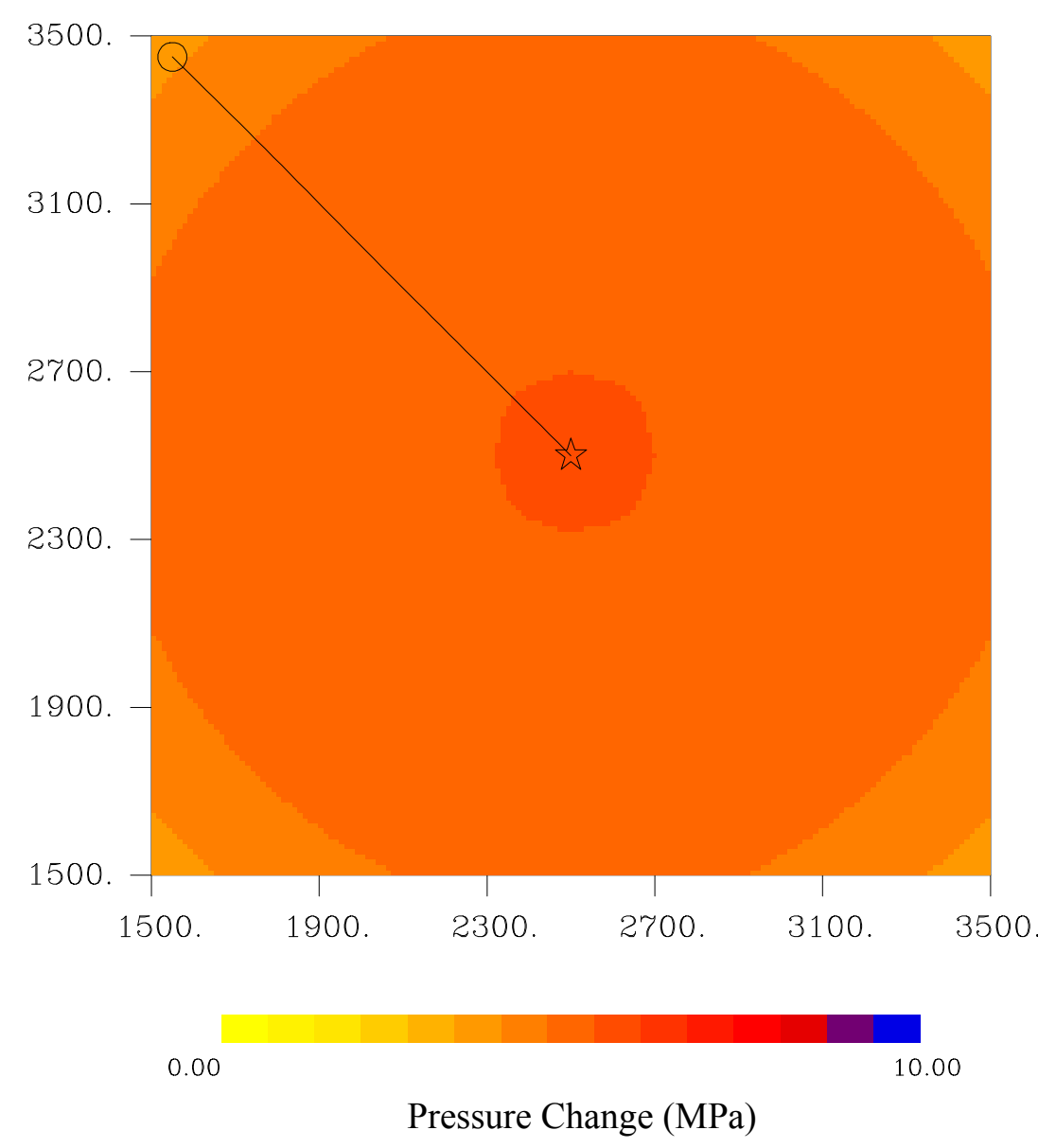

Figure 11. 
Uncoupled

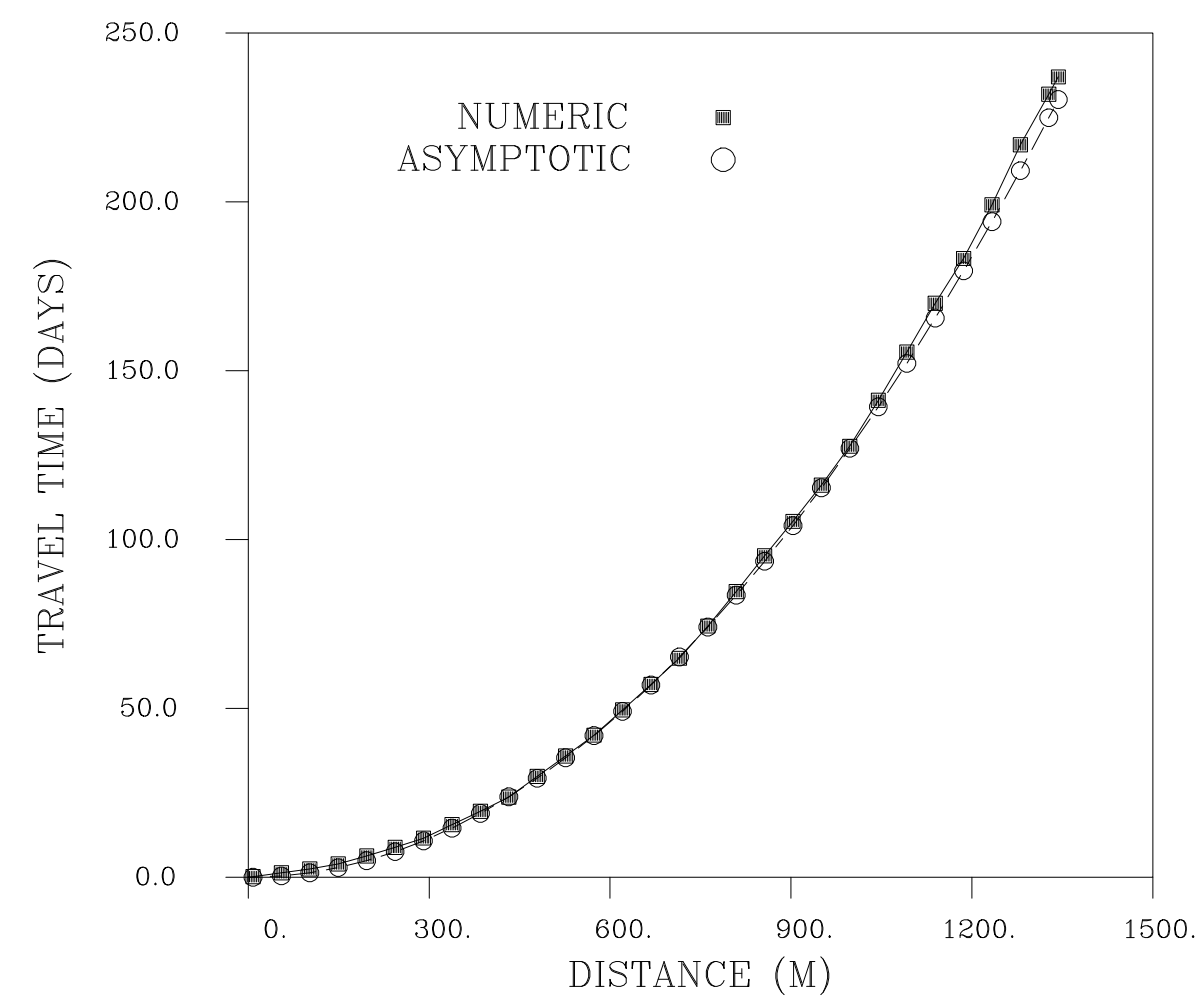

Coupled

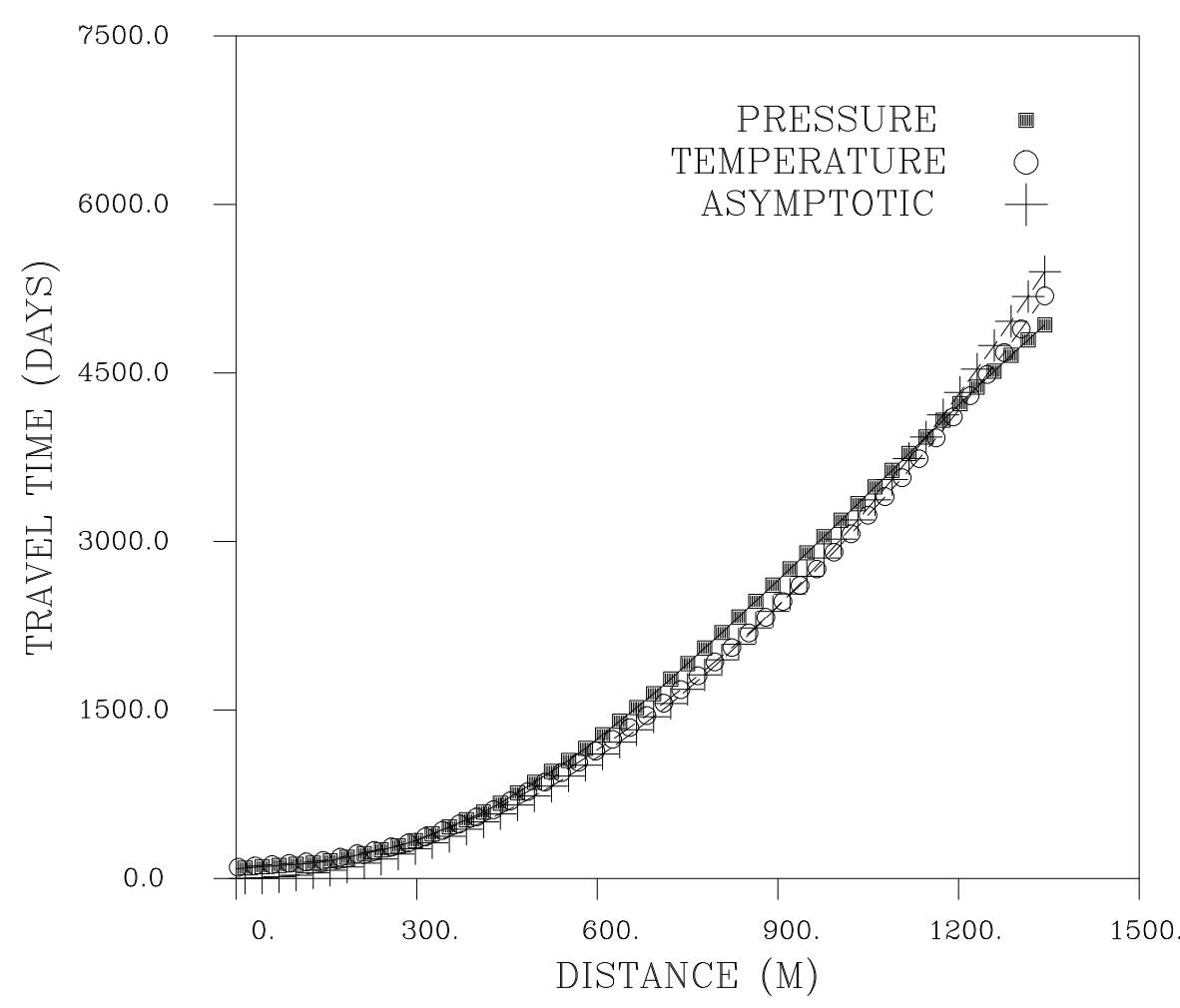

Figure 12 . 


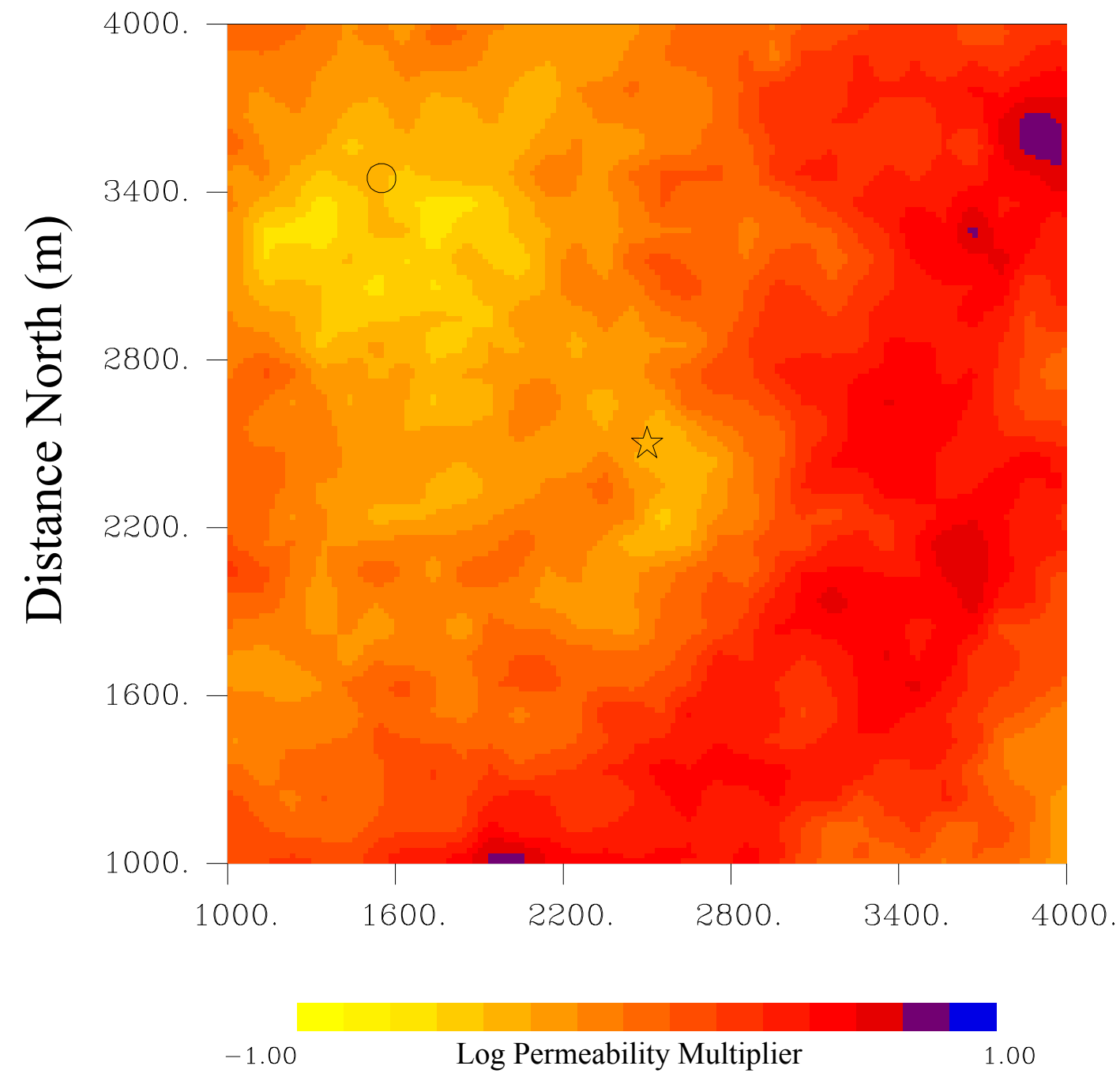

Figure 13. 


\section{DAYS}
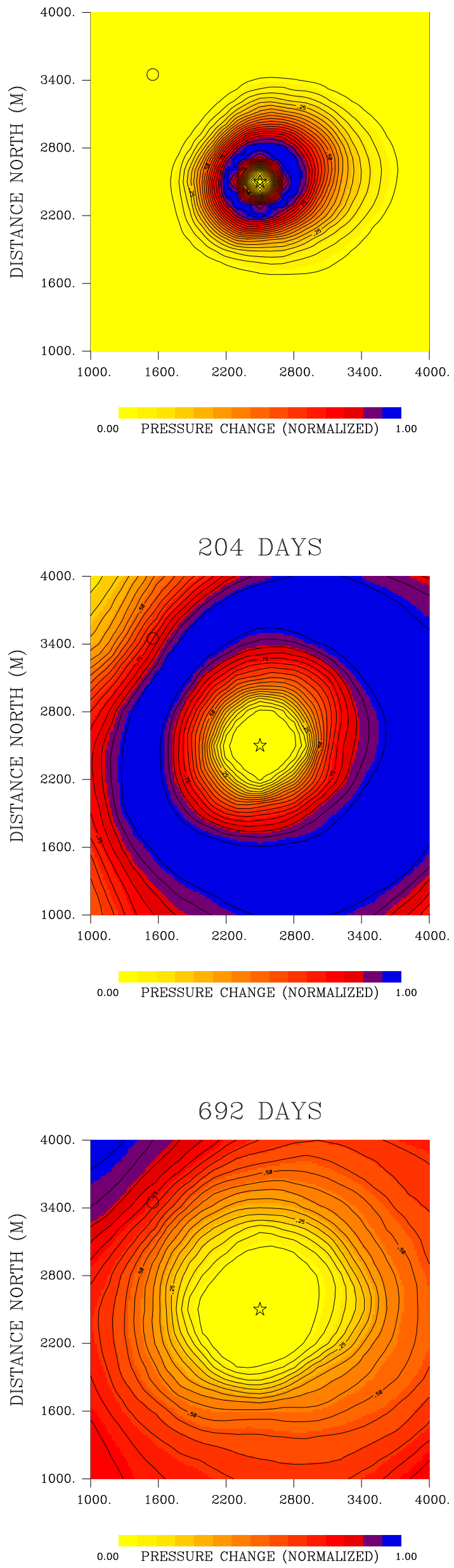


\section{Uncoupled Pressure}

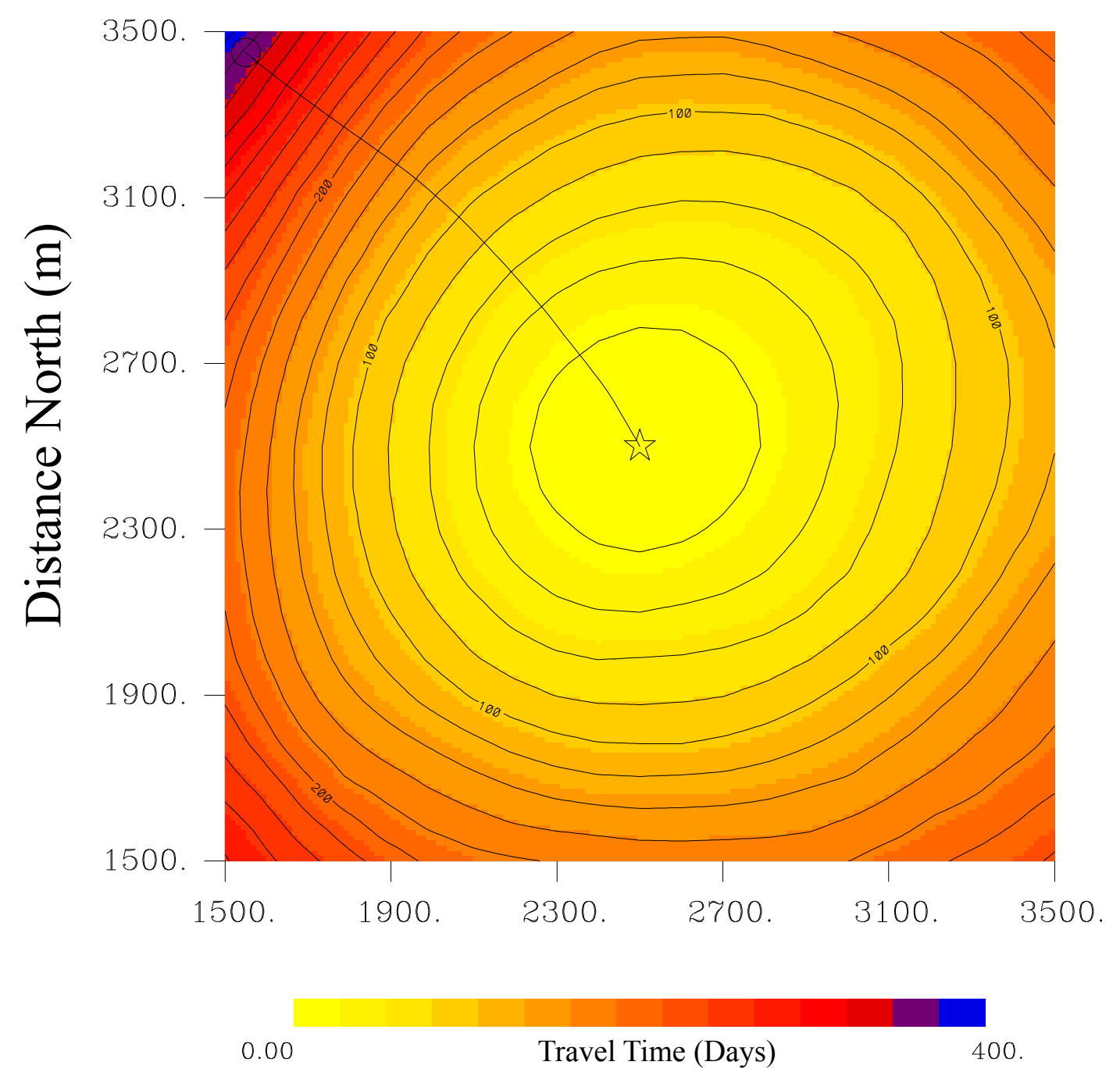

Figure 15. 
367 DAYS

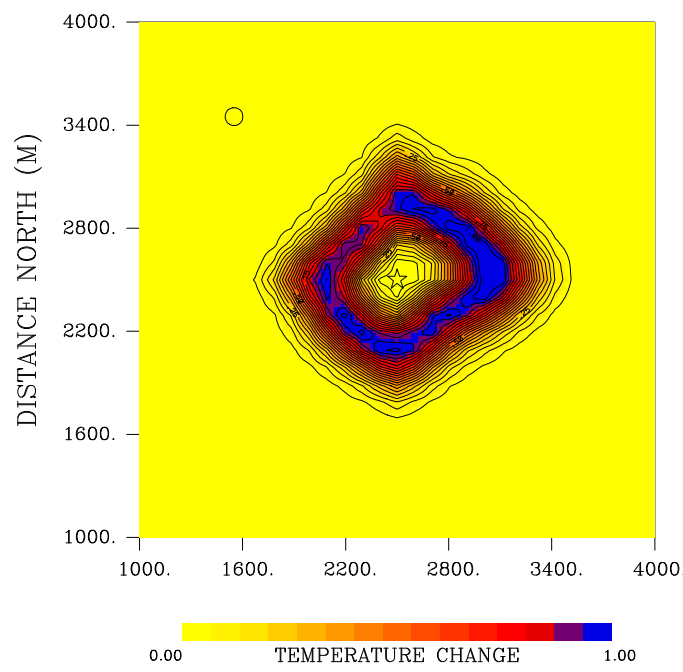

2125 DAYS

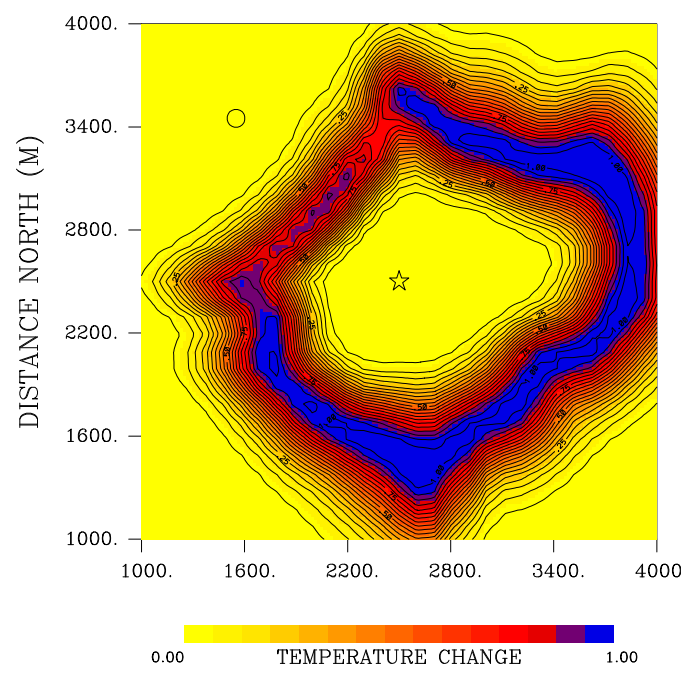

10124 DAYS

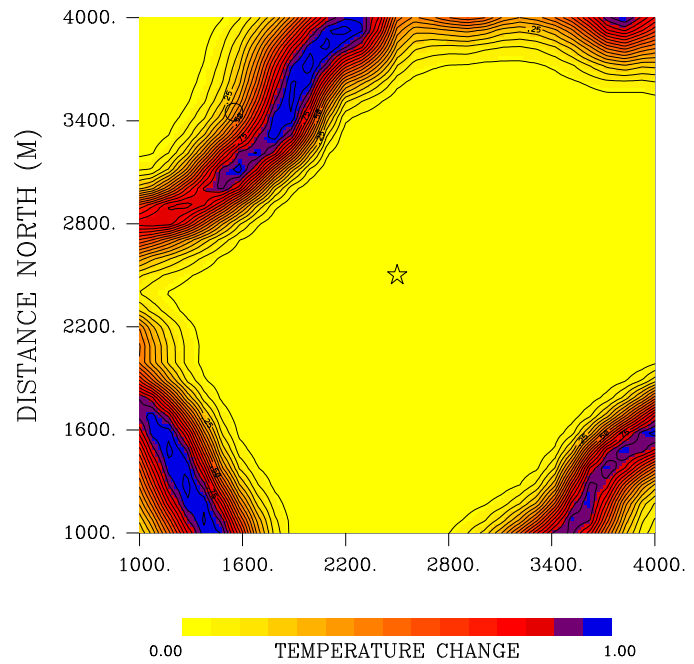

367 DAYS

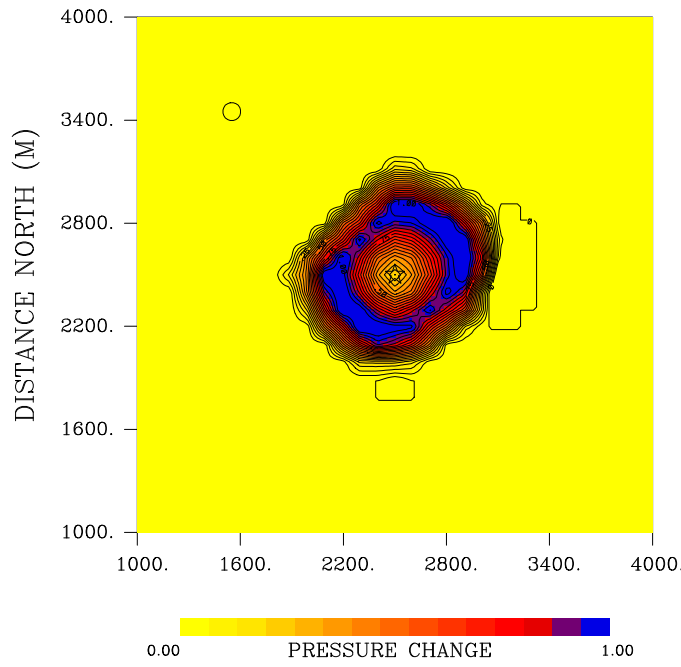

\section{DAYS}

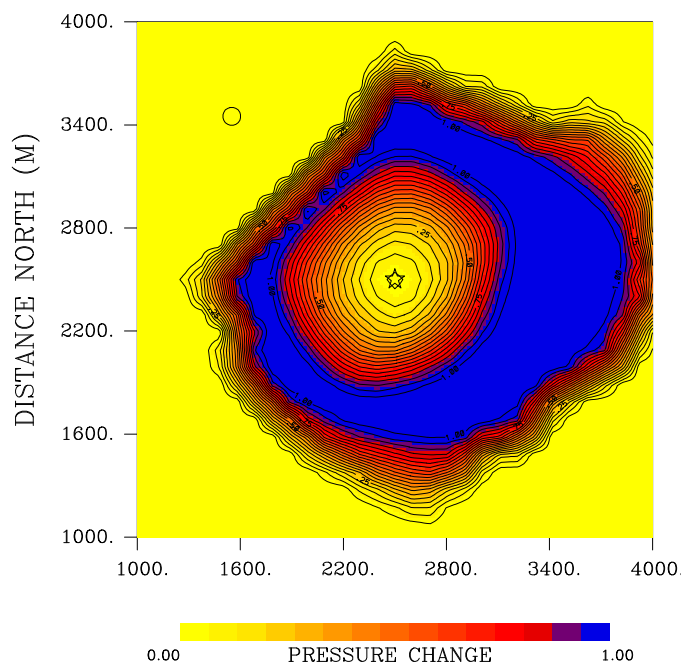

\section{DAYS}

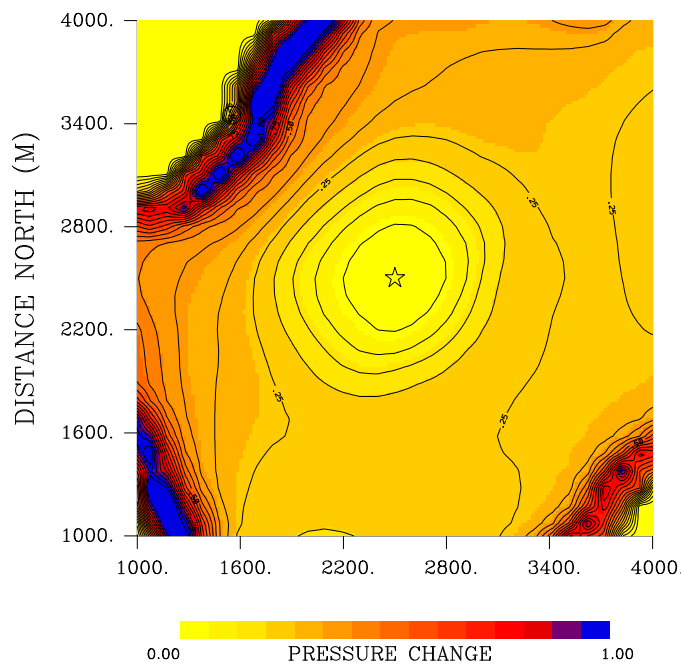




\section{Coupled}

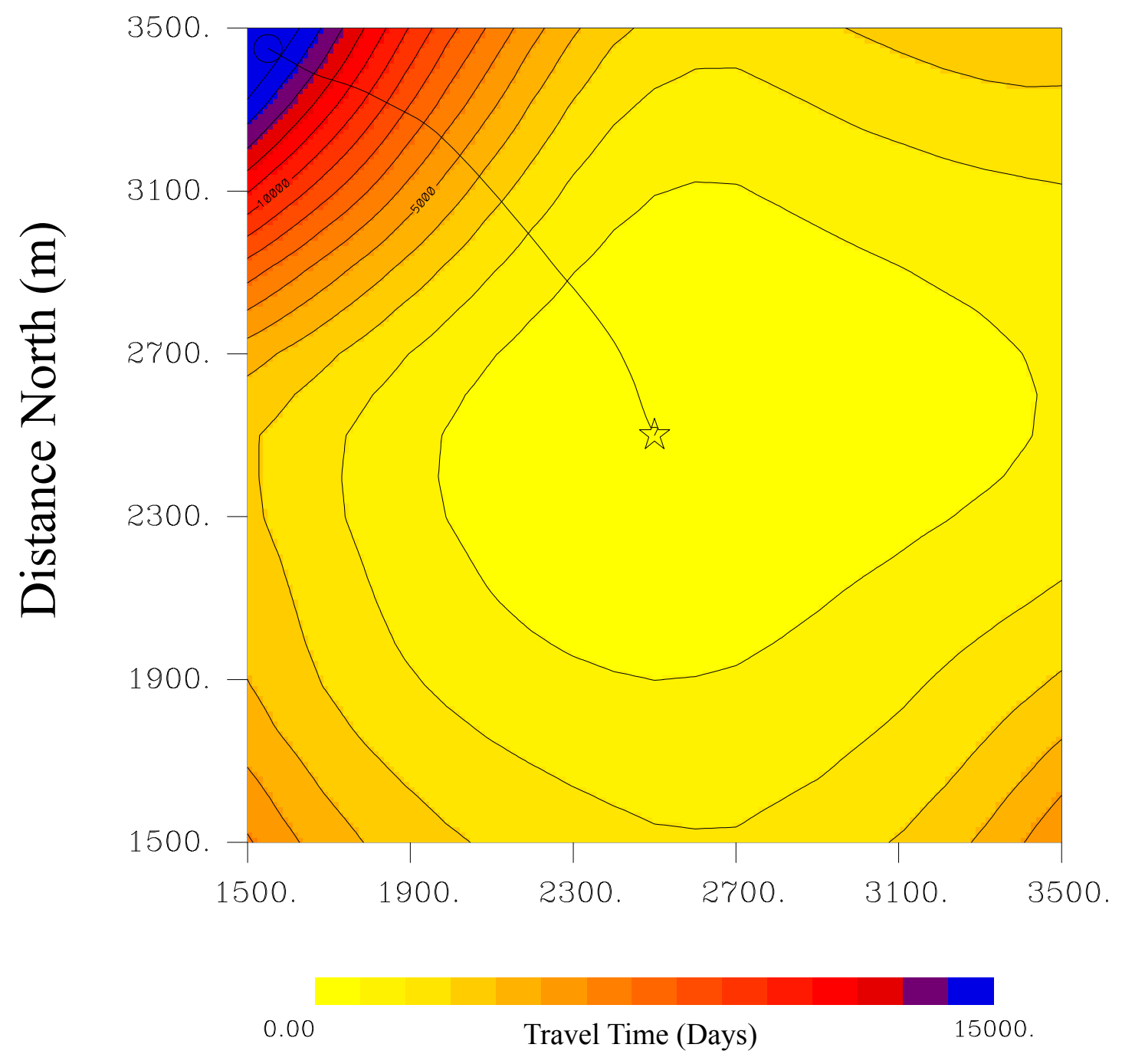

Figure 17. 


\section{Uncoupled}

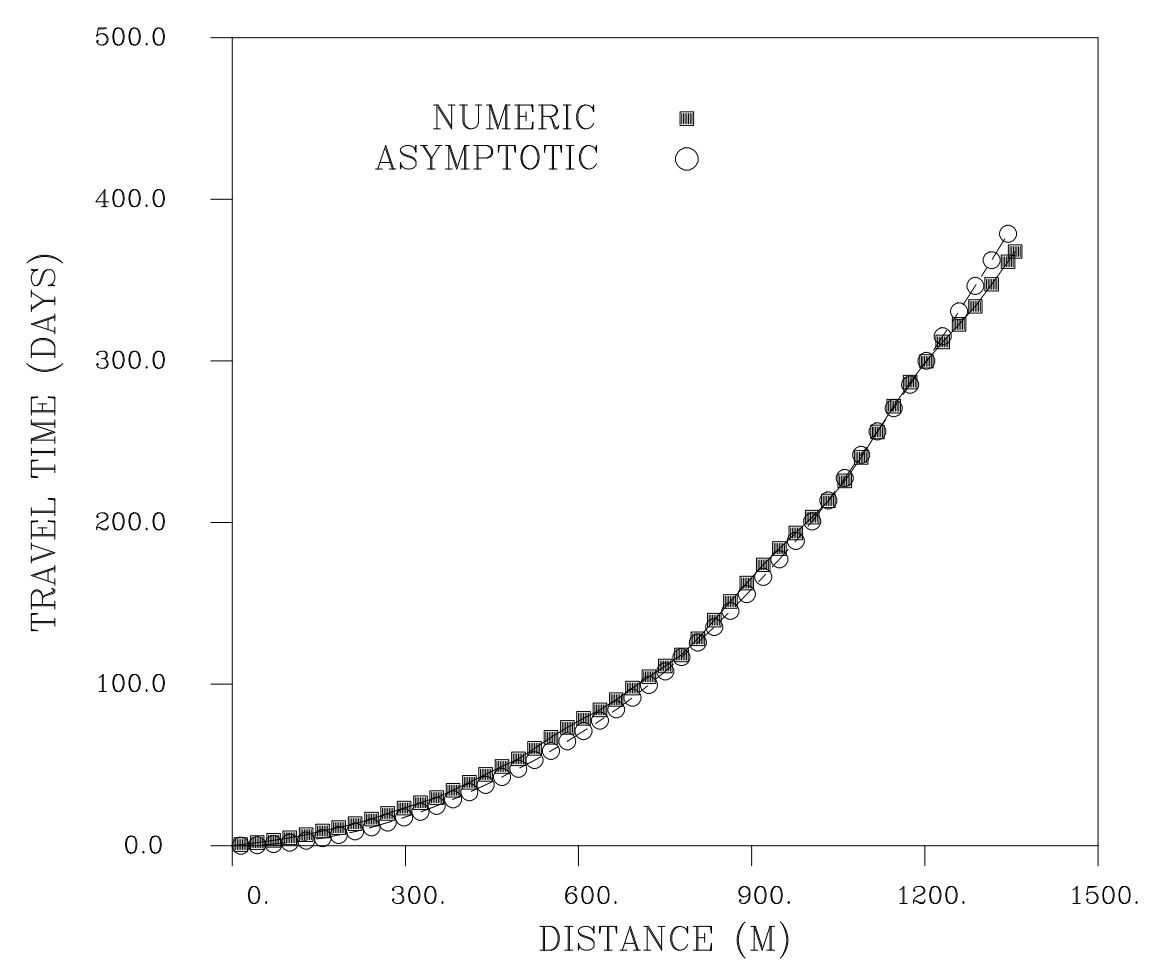

Coupled

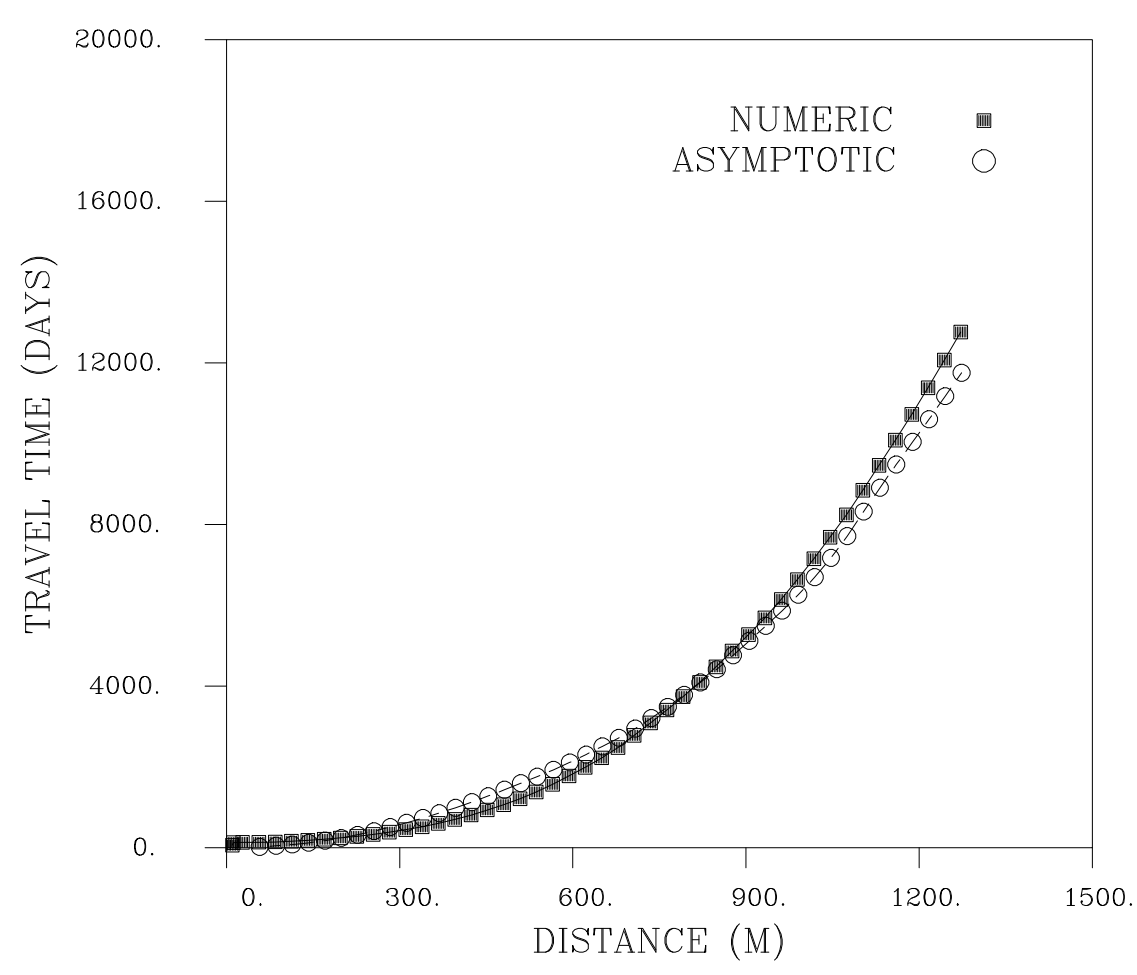

Figure 18. 


\section{DISCLAIMER}

This document was prepared as an account of work sponsored by the United States Government. While this document is believed to contain correct information, neither the United States Government nor any agency thereof, nor The Regents of the University of California, nor any of their employees, makes any warranty, express or implied, or assumes any legal responsibility for the accuracy, completeness, or usefulness of any information, apparatus, product, or process disclosed, or represents that its use would not infringe privately owned rights. Reference herein to any specific commercial product, process, or service by its trade name, trademark, manufacturer, or otherwise, does not necessarily constitute or imply its endorsement, recommendation, or favoring by the United States Government or any agency thereof, or The Regents of the University of California. The views and opinions of authors expressed herein do not necessarily state or reflect those of the United States Government or any agency thereof or The Regents of the University of California.

Ernest Orlando Lawrence Berkeley National Laboratory is an equal opportunity employer. 